\title{
LETTER
}

\section{A likely decade-long sustained tidal disruption}

\section{event}

\author{
Dacheng Lin $^{1}$, James Guillochon ${ }^{2}$, S. Komossa ${ }^{3}$, Enrico Ramirez-Ruiz ${ }^{4}$, Jimmy A. Irwin ${ }^{5,6}$, W. Peter Maksym ${ }^{7}$, Dirk Grupe ${ }^{8}$, \\ Olivier Godet ${ }^{9,10}$, Natalie A. Webb ${ }^{9,10}$, Didier Barret ${ }^{9,10}$, B. Ashley Zauderer ${ }^{11}$, Pierre-Alain Duc ${ }^{12}$, Eleazar R. Carrasco ${ }^{13}$, \\ Stephen D. J. Gwyn ${ }^{14}$
}

\begin{abstract}
${ }^{1}$ Space Science Center, University of New Hampshire, Durham, NH 03824 , USA ${ }^{2}$ Harvard-Smithsonian Center for Astrophysics, The Institute for Theory and Computation, 60 Garden Street, Cambridge, MA 02138, USA ${ }^{3}$ QianNan Normal University for Nationalities, Longshan Street, Duyun City of Guizhou Province, China ${ }^{4}$ Department of Astronomy and Astrophysics, University of California, Santa Cruz, CA 95064, USA ${ }^{5}$ Department of Physics and Astronomy, University of Alabama, Box 870324, Tuscaloosa, AL 35487, USA ${ }^{6}$ Department of Physics and Astronomy, Seoul National University, Seoul 08826, Korea ${ }^{7}$ Harvard-Smithsonian Center for Astrophysics, 60 Garden St., Cambridge, MA 02138, USA ${ }^{8}$ Space Science Center, Morehead State University, 235 Martindale Drive, Morehead, KY 40351, USA ${ }^{9} \mathrm{CNRS}$, IRAP, 9 avenue du Colonel Roche, BP 44346, F-31028 Toulouse Cedex 4, France ${ }^{10}$ Université de Toulouse, UPS-OMP, IRAP, Toulouse, France ${ }^{11}$ Center for Cosmology and Particle Physics, New York University, 4 Washington Place, New York, NY 10003 USA ${ }^{12}$ AIM Paris-Saclay Service d'astrophysique, CEASaclay, 91191 Gif sur Yvette, France ${ }^{13}$ Gemini Observatory/AURA, Southern Operations Center, Casilla 603, La Serena, Chile ${ }^{14}$ Canadian Astronomy Data Centre, Herzberg Institute of Astrophysics, 5071 West Saanich Road, Victoria, British Columbia, V9E 2E7, Canada
\end{abstract}

Multiwavelength flares from tidal disruption and accretion of stars can be used to find and study otherwise dormant massive black holes in galactic nuclei ${ }^{1}$. Previous well-monitored candidate flares are short-lived, with most emission confined to within $\sim 1$ year ${ }^{2.5}$. Here we report the discovery of a well observed super-long ( $>11$ years) luminous soft $X$-ray flare from the nuclear region of a dwarf starburst galaxy. After an apparently fast rise within $\sim 4$ months a decade ago, the $\mathrm{X}$-ray luminosity, though showing a weak trend of decay, has been persistently high at around the Eddington limit (when the radiation pressure balances the gravitational force). The X-ray spectra are generally soft (steeply declining towards higher energies) and can be described with Comptonized emission from an optically thick low-temperature corona, a super-Eddington accretion signature often observed in accreting stellar-mass black holes ${ }^{6}$. Dramatic spectral softening was also caught in one recent observation, implying either a temporary transition from the super-Eddington accretion state to the standard thermal state or the presence of a transient highly blueshifted $(\sim 0.36 \mathrm{c})$ warm absorber. All these properties in concert suggest a tidal disruption event (TDE) of an unusually long super-Eddington accretion phase that has never been observed before.

The X-ray source 3XMM J150052.0+015452 (XJ1500+0154 hereafter) was serendipitously detected in frequent observations ${ }^{7}$ of the foreground galaxy group NGC 5813 by the X-ray observatories Chandra and XMM-Newton from 2005 to 2011. Our follow-up observation of the source with Chandra on February 23rd 2015 provided a well constrained X-ray position coincident with the center of the galaxy SDSS J150052.07+015453.8, to within 0'. 18 (Figure 1, see SI). The galaxy lies at a redshift of 0.145 , or a luminosity distance of $D_{L}=689 \mathrm{Mpc}$ (for $H_{0}=70 \mathrm{~km} \mathrm{~s}^{-1} \mathrm{Mpc}^{-1}, \Omega_{\mathrm{M}}=0.3, \Omega_{\Lambda}=0.7$ ), with strong emission lines indicative of intense star-forming activity. It has a total stellar mass of $\sim 6 \times 10^{9} M_{\odot}$ (see $S I$ ), comparable to that of the Large Magellanic Cloud. For such a small galaxy, we expect ${ }^{8}$ the central supermassive black hole $(\mathrm{SMBH})$ to have mass $\sim 10^{6} M_{\odot}$.

The upper panel in Figure 2 shows the long-term evolution of the $\mathrm{X}$-ray luminosity $L_{\mathrm{X}}$. Our best fits to the spectra with sufficient counts are shown in the lower panel of the figure and are given in Table 1 One striking property of the source is the fast-rise-very-slow-decay outburst profile. It was not detected in the first Chandra observation on April 2nd 2005, with $L_{\mathrm{X}}<4.3 \times 10^{41} \mathrm{erg} \mathrm{s}^{-1}$ ( $3 \sigma$ upper limit, assuming a powerlaw source spectrum of photon index 2.0). Less than 4 months later (July 23rd 2005), the source was detected in the first XMM-Newton observation, with $L_{\mathrm{X}} \sim 5.5 \times 10^{42} \mathrm{erg} \mathrm{s}^{-1}$. It was detected at an even higher luminosity three years later, in one Chandra observation on June 5th 2008 and two XMM-Newton observations in February 2009, with $L_{\mathrm{X}} \sim 7.0 \times 10^{43} \mathrm{erg} \mathrm{s}^{-1}$. The luminosity decreased only slightly to $\sim 3.0 \times 10^{43} \mathrm{erg} \mathrm{s}^{-1}$ in seven Chandra observations in March-April 2011. Similar luminosities were seen in our follow-up observations later, one by Swift on March 28th 2014, one by Chandra on February 23rd 2015, and seven by Swift in February 2016. Given the probably small central SMBH and correcting for emission outside the X-ray band, the source luminosity has been most likely at around the Eddington limit since it went into the outburst.

Another special property of the source is the generally quasi-soft $\mathrm{X}$-ray spectra, most evident in the XMM-Newton and Chandra observations in 2008-2011. These spectra can be roughly described with a dominant thermal disk of apparent maximum disk temperature $k T_{\text {diskbb }} \sim 0.3 \mathrm{keV}$ plus a weak powerlaw (see $S I$ ). However, such a model is physically unacceptable, because the standard thin accretion disk around a $\mathrm{SMBH}$ is expected to produce much cooler thermal emission $\left(k T_{\text {diskbb }} \lesssim 0.1 \mathrm{keV}\right)$. Instead, the spectra can be described well with the Comptonization model CompTT ${ }^{10}$, with an optically thick $(\tau \sim 4-11)$ low-temperature $\left(k T_{\mathrm{e}} \sim 0.4-1.3 \mathrm{keV}\right)$ corona (see $S I$ ). Such spectral parameters are commonly seen in ultraluminous X-ray sources (ULXs) ${ }^{6[11] 12}$, most of which are believed to be super-Eddington accreting stellar-mass black holes, except that XJ1500+0154 had orders of magnitude higher luminosities. Therefore, we identified the observations in 2008-2011 as being in the superEddington accretion state.

Surprisingly, we obtained a much softer X-ray spectrum in the Chandra observation in 2015, mostly due to a drop (by a factor of 7.0) in the count rate above $\sim 1 \mathrm{keV}$ (Figure 2), compared with the observations in 2008-2011. This super-soft spectrum can be described with a dominant thermal disk of $k T_{\text {diskbb }} \sim 0.13 \pm 0.01 \mathrm{keV}$ plus a very weak powerlaw (Figure 2). Such a cool thermal disk is ex- 
pected from accretion onto a SMBH below the Eddington limit. Then the source could be in the thermal state, which in turn supports the identification of the super-Eddington accretion state in previous observations. In this case the X-ray spectral evolution of XJ1500+0154 is very similar to a transient ULX in M3 ${ }^{6}$, which changed from a superEddington accretion state to a thermal state within 20 days with the X-ray luminosity decreasing only slightly. However, we find that the spectrum can be described equally well with the CompTT model that fits the Chandra observations in 2011, except for additional absorption by a strong $\left(N_{\mathrm{H}} \sim 6 \times 10^{23} \mathrm{~cm}^{-2}\right)$ ionized $(\log (\xi)=2.8)$ absorber with a blueshifted velocity of $0.36 c$. Powerful sub-relativistic winds are expected in super-Eddington accreting black holes 13 , and highly blueshifted warm absorbers have been detected in ULX $\$$ 14 . Therefore, this interpretation for the Chandra observation in 2015 also supports the identification of the super-Eddington accretion state in previous observations. The recent Swift observations in 2016 have poor statistics, but some of them did not seem to show similar super-soft X-ray spectra. Therefore, the source has not completely settled to a new state of super-soft X-ray spectra, and the super-Eddington accretion seems to have lasted for $\gtrsim 11$ years (see $S I$ ).

No sign of persistent nuclear activity is seen in the optical emission lines of the host galaxy, whose ratios are fully consistent with those expected from a starburst galaxy. There are many other properties of the source that argue against the possibility that it is a standard active galactic nucleus (AGN, see $S I$ ). In particular, no AGN is known to show X-ray spectra as soft as XJ1500+0154 within the $1-4.5 \mathrm{keV}$ energy band or show dramatic quasi-soft to super-soft X-ray spectral change. The large X-ray variability (a factor of $>97$ ) is also extremely rare among AGNs. Therefore, although we cannot completely rule out that $\mathrm{XJ} 1500+0154$ is just a highly variable AGN at this point, its Xray outburst is best explained as tidal disruption of a star by the central black hole. This interpretation is strongly supported by our new discovery of two other sources that seemed to be in X-ray outbursts with similar quasi-soft X-ray spectra as XJ1500+0154 but have host galaxies showing no sign of nuclear activity in optical (see $S I$ ).

The super-Eddington accretion phase from tidal disruption of a solar-type star by a $10^{6} M_{\odot}$ black hole can last $t_{\mathrm{Edd}} \approx 2$ years, with the peak mass accretion rate highly super-Eddingtor $1 / 15$. One main property of a super-Eddington accretion disk is a lower radiative efficiency than a standard thermal thin disk, due to significant superEddington effects of photon trapping and outflows in the inner disk regior $13 / 16 / 17$. These effects are more serious at higher accretion rates, with the disk luminosity sustained at around the Eddington limit. The Eddington-limited slow decay of our source thus agrees well with the super-Eddington accretion signatures suggested by the X-ray spectra. The long super-Eddington accretion phase of $\gtrsim 11$ years in our event would imply disruption of a very massive star $\left(10 M_{\odot}\right)$ based on the standard theory 15 . However, it has been realized that the evolution of TDEs heavily depends on how the streams of tidal debris intersect each other ${ }^{[1822]}$. It should be common for circularization of the fallback mass onto the accretion disk to occur at a much larger distance, resulting in a much longer viscous time scale, than predicted from the standard theory $\sqrt{19}$. Therefore, $t_{\mathrm{Edd}}$ can be very long in a slow circularization process, unless the circularization is so slow that the peak accretion rate drops to be sub-Eddington.

We plot in Figure 2 (solid line) the evolution of the luminosity from a full disruption of a $2 M_{\odot}$ star by a $10^{6} M_{\odot}$ black hole, with the accretion of the mass slowed relative to the fallback time by 3 years. The super-Eddington effects were taken into account by introducing a logarithmic dependence of the radiative efficiency on the accretion rate above the Eddington limit ${ }^{13}$ (see $S I$ ). We assumed that $25 \%$ of the radiation is in X-rays, as inferred from the spectral modeling. The model describes the data well. The total energy release and the total mass accreted onto the black hole until the last Swift observation would then be $6.4 \times 10^{52} \mathrm{ergs}$ and $0.89 M_{\odot}$, respectively, which are orders of magnitude higher than seen in other known events 5 [23,24.

Although disruption of a very massive star of $10 M_{\odot}$ with prompt circularization can also describe the data, such disruption is expected to be orders of magnitude rarer than disrupting a star of $2 M_{\odot}$ with slow circularization (see $S I$ ). Therefore our event provides the first convincing evidence of slow circularization effects in TDEs, which are expected to be very common when the black holes are small $\left(\sim 10^{6}\right.$ $M_{\odot}$ ) but were not clearly observed before probably due to observational bias 19

We calculated the rate of events similar to XJ1500+0154 to be $\sim 4 \times 10^{-7}$ per galaxy per year (see $S I$ ), which is about two orders of magnitude lower than estimated for short TDEs ${ }^{25}$. One main reason for the low rate of events like XJ1500+0154 could be the relatively large mass $\left(2 M_{\odot}\right)$ of the disrupted stars required, which is only possible in starburst galaxies ${ }^{26}$. Although events like XJ1500+0154 are rare, their extreme duration and radiative inefficiency mean that their contributions to the luminosity function of active galactic nuclei and to the growth of the black holes are comparable to or even higher than those of short events. TDEs with a shorter super-Eddington accretion phase than XJ1500+0154 could be more common. The discovery of our event opens up a new realm in which to search for super-Eddington accreting TDEs, that is, by investigating sources with quasi-soft X-ray spectra. Our discovery of the other two candidates is the result of applying this scheme.

This is the first time that X-ray spectra resembling typical superEddington accreting stellar-mass black holes were observed in an accreting SMBH. If our interpretation of a super-Eddington accreting TDE for XJ1500+0154 is correct, it would have important implications for the growth of massive black holes. The detection of quasars at redshift $z>6$ with black hole masses $\sim 10^{9} M_{\odot}$ poses a problem to explain their growth with accretion via a standard thin disk at the Eddington rate ${ }^{27}$. However, their formation would be possible if the black holes can accrete at a super-Eddington rate during an early phase $e^{28}$. Our event shows that super-Eddington accretion onto massive black holes can occur, giving strong observational support to this model. The high absorption in these systems would mean that the search for them should be through radio and infrared ${ }^{29}$, because their X-ray spectra, if as soft as XJ1500+0154, would be completely absorbed.

We expect the accretion rate to drop by an order of magnitude to be well sub-Eddington in the next ten years based on our model of full disruption of a $2 M_{\odot}$ star. By continued monitoring of the event, we will be able to test our TDE interpretation and to determine the duration of the super-Eddington accretion phase and the origin of spectral softening. We will also be given a rare opportunity to observe the spectral evolution of the event across different accretion regimes and to investigate its connection with short super-soft events that are mostly believed to accrete below the Eddington limit.

1. Rees, M. J. Tidal disruption of stars by black holes of 10 to the 6 th- 10 to the 8th solar masses in nearby galaxies. Nature 333, 523-528 (1988).

2. Gezari, S. et al. An ultraviolet-optical flare from the tidal disruption of a helium-rich stellar core. Nature 485, 217-220 (2012). 1205.0252

3. Zauderer, B. A. et al. Radio Monitoring of the Tidal Disruption Event Swift J164449.3+573451. II. The Relativistic Jet Shuts Off and a Transition to Forward Shock X-Ray/Radio Emission. Astrophys. J. 767, 152 (2013). 1212 .1173

4. Miller, J. M. et al. Flows of X-ray gas reveal the disruption of a star by a massive black hole. Nature 526, 542-545 (2015). 1510.06348

5. van Velzen, S. et al. A radio jet from the optical and $\mathrm{x}$-ray bright stellar tidal disruption flare ASASSN-14li. Science 351, 62-65 (2016). 1511.08803 
6. Middleton, M. J. et al. Bright radio emission from an ultraluminous stellar-mass microquasar in M 31. Nature 493, 187-190 (2013). 1212.4698

7. Randall, S. W. et al. A Very Deep Chandra Observation of the Galaxy Group NGC 5813: AGN Shocks, Feedback, and Outburst History. Astrophys. J. 805, 112 (2015). 1503.08205

8. Reines, A. E. \& Volonteri, M. Relations between Central Black Hole Mass and Total Galaxy Stellar Mass in the Local Universe. Astrophys. J. 813, 82 (2015). 1508.06274

9. Gierliński, M. \& Done, C. Is the soft excess in active galactic nuclei real? Mon. Not. R. Astron. Soc. 349, L7-L11 (2004). arXiv:astro-ph/0312271

10. Titarchuk, L. Generalized Comptonization models and application to the recent high-energy observations. Astrophys. J. 434, 570-586 (1994).

11. Gladstone, J. C., Roberts, T. P. \& Done, C. The ultraluminous state. Mon. Not. R. Astron. Soc. 397, 1836-1851 (2009). 0905.4076

12. Lin, D., Irwin, J. A., Webb, N. A., Barret, D. \& Remillard, R. A. Discovery of a Highly Variable Dipping Ultraluminous X-Ray Source in M94. Astrophys. J. 779, 149 (2013). 1311.1198

13. King, A. \& Muldrew, S. I. Black hole winds II: Hyper-Eddington winds and feedback. Mon. Not. R. Astron. Soc. 455, 1211-1217 (2016). 1510.01736

14. Pinto, C., Middleton, M. J. \& Fabian, A. C. Resolved atomic lines reveal outflows in two ultraluminous X-ray sources. Nature (2016). 1604.08593

15. Ulmer, A. Flares from the Tidal Disruption of Stars by Massive Black Holes. Astrophys. J. 514, 180-187 (1999).

16. Ohsuga, K. \& Mineshige, S. Why Is Supercritical Disk Accretion Feasible? Astrophys. J. 670, 1283-1290 (2007). arXiv: 0710.2941

17. Krolik, J. H. \& Piran, T. Jets from Tidal Disruptions of Stars by Black Holes. Astrophys. J. 749, 92 (2012). 1111.2802

18. Kochanek, C. S. The aftermath of tidal disruption: The dynamics of thin gas streams. Astrophys. J. 422, 508-520 (1994).

19. Guillochon, J. \& Ramirez-Ruiz, E. A Dark Year for Tidal Disruption Events. Astrophys. J. 809, 166 (2015). 1501.05306

20. Piran, T., Svirski, G., Krolik, J., Cheng, R. M. \& Shiokawa, H. Disk Formation Versus Disk Accretion: What Powers Tidal Disruption Events? Astrophys. J. 806, 164 (2015). 1502.05792

21. Shiokawa, H., Krolik, J. H., Cheng, R. M., Piran, T. \& Noble, S. C. General Relativistic Hydrodynamic Simulation of Accretion Flow from a Stellar Tidal Disruption. Astrophys. J. 804, 85 (2015). 1501.04365

22. Hayasaki, K., Stone, N. \& Loeb, A. Circularization of tidally disrupted stars around spinning supermassive black holes. Mon. Not. R. Astron. Soc. 461, 3760-3780 (2016). 1501.05207

23. Li, L.-X., Narayan, R. \& Menou, K. The Giant X-Ray Flare of NGC 5905 Tidal Disruption of a Star, a Brown Dwarf, or a Planet? Astrophys. J. 576, 753-761 (2002). astro-ph/0203191

24. Komossa, S. et al. A Huge Drop in the X-Ray Luminosity of the Nonactive Galaxy RX J1242.6-1119A, and the First Postflare Spectrum: Testing the Tidal Disruption Scenario. Astrophys. J. 603, L17-L20 (2004). arXiv:astro-ph/0402468

25. Donley, J. L., Brandt, W. N., Eracleous, M. \& Boller, T. LargeAmplitude X-Ray Outbursts from Galactic Nuclei: A Systematic Survey using ROSAT Archival Data. Astron. J. 124, 1308-1321 (2002). astro-ph/0206291

26. Kochanek, C. S. Tidal disruption event demographics. Mon. Not. R. As tron. Soc. 461, 371-384 (2016). 1601.06787

27. Mortlock, D. J. et al. A luminous quasar at a redshift of $z=7.085$. Nature 474, 616-619 (2011). 1106.6088

28. Volonteri, M. \& Rees, M. J. Rapid Growth of High-Redshift Black Holes. Astrophys. J. 633, 624-629 (2005). astro-ph/0506040

29. Martínez-Sansigre, A. et al. The obscuration by dust of most of the growth of supermassive black holes. Nature 436, 666-669 (2005). astro-ph/0505486

30. Maksym, W. P., Ulmer, M. P., Eracleous, M. C., Guennou, L. \& Ho, L. C. A tidal flare candidate in Abell 1795. Mon. Not. R. Astron. Soc. 435 , 1904-1927 (2013). 1307.6556

Acknowledgments D.L. is supported by the National Aeronautics and Space Administration through Chandra Award Number GO5-16087X issued by the Chandra X-ray Observatory Center, which is operated by the Smithsonian Astrophysical Observatory for and on behalf of the National Aeronautics Space Administration under contract NAS8-03060. We thank the Swift PI Neil Gehrels for approving our ToO request to make several observations of XJ1500+0154.
Author Contributions D.L. wrote the main manuscript and led the data analysis. J. G. helped with the modeling of the long-term X-ray light curve and wrote the text on the modeling in the Supplementary Information. S. G. stacked the CFHT images. All authors discussed the results and commented on the manuscript.

Competing Interests The authors declare that they have no competing financial interests.

Author Information Correspondence and requests for materials should be addressed to D.L. (dacheng.lin@unh.edu). 
Table 1 | Spectral fit results for high-quality spectra. The X1 and C10 spectra were rebinned to have at least one count per bin and were fitted by minimizing the $\mathrm{C}$ statistic, while the $\mathrm{X} 2, \mathrm{X} 3, \mathrm{C} 2$, and $\mathrm{C} 3-\mathrm{C} 9$ spectra were rebinned to have at least 20 counts per bin and were fitted by minimizing the $\chi^{2}$ statistic. The fits used energy channels within $0.3-10 \mathrm{keV}$ for XMM-Newton and energy channels within $0.3-7 \mathrm{keV}$ for Chandra. All models include Galactic absorption of column density $N_{\mathrm{H}, \mathrm{Gal}}=4.4 \times 10^{20} \mathrm{~cm}^{-2}$. The absorption intrinsic to the X-ray source at redshift 0.14542 was also included and fixed at $N_{\mathrm{H}, \mathrm{i}}=4.2 \times 10^{21} \mathrm{~cm}^{-2}$, which was the best-fitting value from the simultaneous fit to the $\mathrm{C} 2, \mathrm{X} 3$, and $\mathrm{C} 3-\mathrm{C} 9$ spectra. $L_{\mathrm{abs}}$ is the source rest-frame $0.34-11.5 \mathrm{keV}$ luminosity, corrected for the Galactic absorption but not intrinsic absorption, and $L_{\text {unabs }}$ is the source rest-frame $0.34-11.5 \mathrm{keV}$ luminosity, corrected for both Galactic and intrinsic absorption. Both $L_{\text {abs }}$ and $L_{\text {unabs }}$ are in units of $10^{43} \mathrm{erg} \mathrm{s}^{-1}$. All errors are at the $90 \%$-confidence level. Parameters without errors were fixed in the fits. For C10, two models were tested: diskbb+PL and zxipcf(comptt). For the latter model, the CompTT component was fixed at the best fit of this model to C3-C9, and the luminosities $L_{\text {abs }}$ and $L_{\text {unabs }}$ was simply copied from those of $\mathrm{C} 3-\mathrm{C} 9$. The reduced $\chi^{2}$ values are given for fits using the $\chi^{2}$ statistic, but not for those using the $\mathrm{C}$ statistic.

\begin{tabular}{|c|c|c|c|c|c|}
\hline Obs. & Model & Parameters & $\chi_{\nu}^{2}(\nu)$ & $L_{\mathrm{abs}}$ & $L_{\text {unabs }}$ \\
\hline $\mathrm{X} 1$ & diskbb+PL & $\begin{array}{c}k T_{\text {diskbb }}=0.10_{-0.04}^{+0.06} \mathrm{keV}, N_{\text {diskbb }}=210_{-204}^{+27841} \\
\Gamma_{\mathrm{PL}}=2.5, N_{\mathrm{PL}}=4.6_{-3.4}^{+3.9} \times 10^{-6}\end{array}$ & $\cdots$ & $0.08_{-0.03}^{+0.04}$ & $0.55_{-0.31}^{+0.95}$ \\
\hline $\mathrm{X} 2$ & CompTT & $k T_{0}=0.04 \mathrm{keV}, k T_{\mathrm{e}}=0.35_{-0.05}^{+0.08} \mathrm{keV}, \tau=10.8_{-2.1}^{+2.4}$ & $1.09(106)$ & $1.16_{-0.06}^{+0.06}$ & $6.18_{-0.60}^{+0.65}$ \\
\hline X3 & CompTT & $k T_{0}=0.04 \mathrm{keV}, k T_{\mathrm{e}}=0.51_{-0.13}^{+0.30} \mathrm{keV}, \tau=7.3_{-2.2}^{+2.1}$ & $0.90(99)$ & $1.13_{-0.06}^{+0.06}$ & $7.03_{-0.66}^{+0.71}$ \\
\hline $\mathrm{C} 2$ & CompTT & $k T_{0}=0.04 \mathrm{keV}, k T_{\mathrm{e}}=0.51_{-0.14}^{+0.33} \mathrm{keV}, \tau=7.6_{-2.5}^{+3.0}$ & $0.90(43)$ & $1.08_{-0.09}^{+0.09}$ & $6.24_{-1.32}^{+1.55}$ \\
\hline C3-C9 & CompTT & $k T_{0}=0.04 \mathrm{keV}, k T_{\mathrm{e}}=1.33_{-0.43}^{+1.48} \mathrm{keV}, \tau=3.9_{-1.7}^{+1.3}$ & $0.83(138)$ & $0.60_{-0.02}^{+0.02}$ & $3.38_{-0.21}^{+0.23}$ \\
\hline \multirow[t]{2}{*}{ C10 } & diskbb+PL & $\begin{array}{c}k T_{\text {diskbb }}=0.13_{-0.01}^{+0.01} \mathrm{keV}, N_{\text {diskbb }}=312_{-154}^{+310} \\
\Gamma_{\mathrm{PL}}=2.5, N_{\mathrm{PL}}=2.2_{-1.2}^{+1.7} \times 10^{-6}\end{array}$ & $\cdots$ & $0.31_{-0.04}^{+0.05}$ & $2.88_{-0.66}^{+0.86}$ \\
\hline & & $\begin{array}{c}k T_{0}=0.04 \mathrm{keV}, k T_{\mathrm{e}}=1.33 \mathrm{keV}, \tau=3.9, \\
N_{\mathrm{H}}=64 \pm 10 \times 10^{22} \mathrm{~cm}^{-2}, \log (\xi)=2.78 \pm 0.04, z=-0.36 \pm 0.02\end{array}$ & $\cdots$ & $0.60_{-0.02}^{+0.02}$ & $3.38_{-0.21}^{+0.23}$ \\
\hline
\end{tabular}




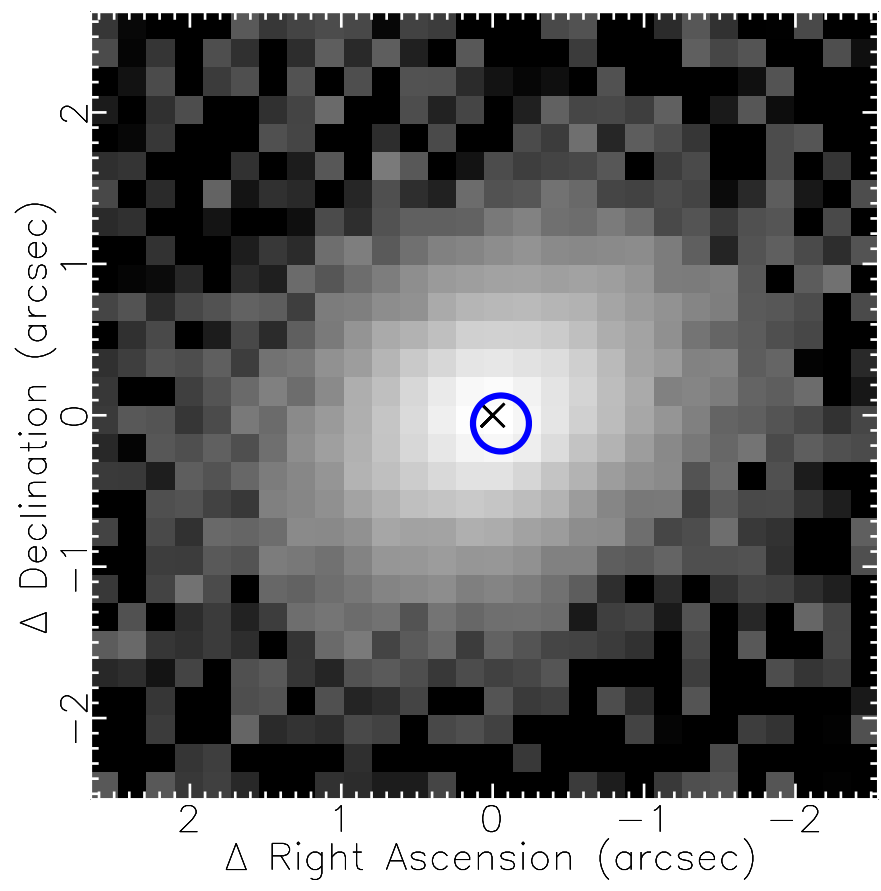

Figure 1 The CFHT/MegaPrime $r^{\prime}$-band image around the field of XJ1500+0154 indicates its galactic nuclear origin. The origin of the image is at the center of the galaxy SDSS J150052.07+015453.8 (black cross). The blue circle of radius 0. ' 18 ( $0.5 \mathrm{kpc}$ ) represents the $95 \%$ positional uncertainty of XJ1500+0154. 

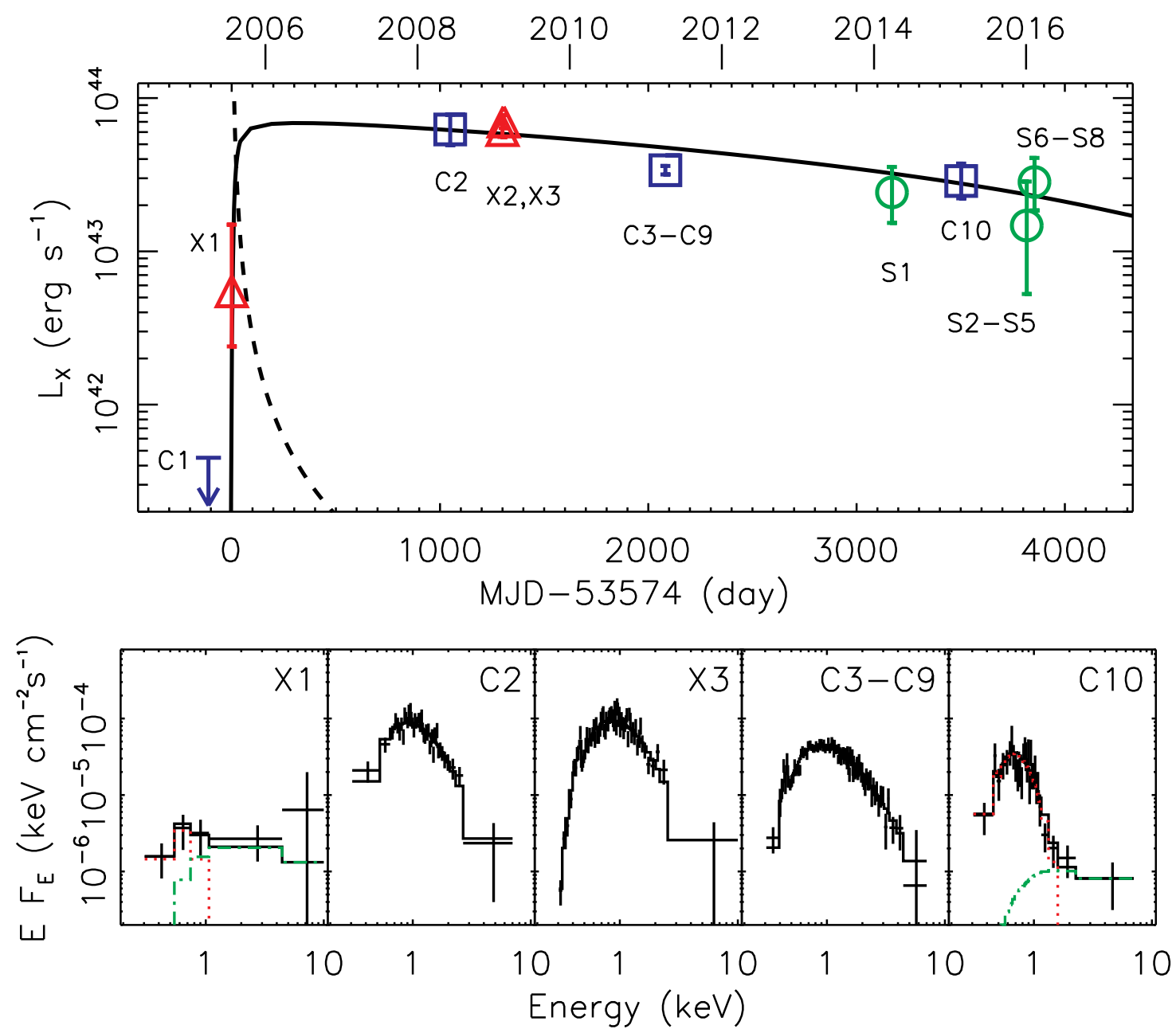

Figure 2 | The long-term evolution of the X-ray luminosity and spectrum of XJ1500+0154. Upper panel: The long-term source rest-frame 0.34-11.5 keV unabsorbed luminosity curve. The Chandra, XMM-Newton and Swift observations are shown as blue squares, red triangles and green circles, respectively, with $90 \%$ error bars, but for the first Chandra observation $\mathrm{C} 1$ in 2005 the $3 \sigma$ upper limit is shown with an arrow. We have merged the seven Chandra observations in 2011 to create a single coadded spectrum, given the lack of significant spectral/flux change in these observations. Similarly we also created a coadded spectrum from the combination of S2-S5 and another one from S6-S8. For clarity, we have offset S2-S5 to be one month earlier, because they are too close to S6-S8 in time. The solid line is a model of disrupting a $2 M \odot$ star by a black hole of mass $10^{6} M \odot$ with slow circularization and super-Eddington effects (see $S I$ ). Such a model describes the data well. The dashed line plots $t^{-5 / 3}$, assuming a peak X-ray luminosity of $10^{44} \mathrm{erg} \mathrm{s}^{-1}$ that is reached two months after disruption of the star; it represents the typical evolution trend for thermal TDEs 24,30 , which obviously last much shorter than our event. Lower panels: The unfolded X-ray spectra. The X1 and C10 observations were fitted with a diskbb model (red dotted line) plus a PL (green dot-dashed line), and the C2, X2, X3, and C3-C9 observations were fitted with a CompTT model (the X2 spectrum is not shown but looks very similar to X3). Note that C10 can also be described with the CompTT fit to C3-C9 subject to a fast warm absorber. For clarity, we show only pn data for the XMM-Newton observations. Also for clarity, the spectra were rebinned to be above $2 \sigma$ in each bin in the plot. 


\section{Method}

XMM-Newton observations and data analysis

$\mathrm{XJ} 1500+0154$ was serendipitously detected at off-axis angles of $\sim 13^{\prime}$ in three XMM-Newton observations (X1-X3 hereafter; see Supplementary Table 1) of NGC 5813. X1 was made in July 2005 , while X2 and X3 were made in February 2009, only six days apart. The source was detected in all three European Photon Imaging Cameras (i.e., pn, MOS1/M1, and MOS2/M2 $\sqrt{31.33}$ in the imaging mode in $\mathrm{X} 1$, but only in pn and MOS2 in $\mathrm{X} 2$ and $\mathrm{X} 3$ because the source is outside the field of view (FOV) of MOS1 in these two observations. We used SAS 15.0.0 and the calibration files of 2016 February for reprocessing the X-ray event files and follow-up analysis. The data in strong background flare intervals, seen in all cameras in all observations, were excluded following the SAS thread for the filtering against high backgrounds. The final clean exposures used are given in Supplementary Table 1. We extracted the source spectra from all available cameras using a circular region of radius $20^{\prime \prime}$. The background spectra were extracted from a large circular region near the source, using a radius of $100^{\prime \prime}$ for MOS 1 and MOS2 and a radius of $50^{\prime \prime}$ for pn. The event selection criteria followed the default values in the pipeline ${ }^{34}$. For $\mathrm{X} 2$ and $\mathrm{X} 3$, in which the source was bright, we also extracted the pn light curves binned at $500 \mathrm{~s}$ using the SAS tool epiclccorr and performed variability tests using the ekstest tool. We used the $0.3-3 \mathrm{keV}$ band, where the source counts dominated over those of the background.

\section{Chandra observations and data analysis}

XJ1500+0154 was serendipitously covered in nine Chandra observations (C1-C9 hereafter; see Supplementary Table 1) of NGC 5813, but all at large pointing offsets $\left(11^{\prime}-15^{\prime}\right)$. The dense observations in 2011 (C3-C9, 0.5 Ms) are from a Large Program (LP, PI: Dr. Scott Randall) on NGC 5813. All nine observations used the imaging array of the AXAF CCD Imaging Spectrometer (ACIS) ${ }^{35}$, and XJ1500+0154 fell in the back-illuminated chip $\mathrm{S} 1$ in all observations except $\mathrm{C} 2$, in which it fell in the front-illuminated chip I3. We had a Chandra followup observation of the source for $37 \mathrm{ks}$ in February 2015 (C10 hereafter), with the aim point at the back-illuminated chip S3. We reprocessed all the data to apply the latest calibration (CALDB 4.6.7) using the script chandra_repro in the Chandra Interactive Analysis of Observations (CIAO, version 4.7) package. No clear background flares were seen, and we used all data for all observations.

The spectra of XJ1500+0154 were extracted for each observation. We used a circular source region enclosing $90 \%$ of the point spread function (PSF) at $1.0 \mathrm{keV}$ and a circular background region of a radius of $50^{\prime \prime}$ near the source. However, there are three exceptions. For observations $\mathrm{C} 1$ and $\mathrm{C} 2$, we used the $70 \%$ PSF radius for the source region, considering that the source was not detected in $\mathrm{C} 1$ and was near the $\mathrm{CCD}$ edge in $\mathrm{C} 2$. For our follow-up observation $\mathrm{C} 10$, in which the source is near the aim point with minimum background contamination, we used the 95\% PSF radius for the source region. We used the CIAO task mkacisrmf to create the response matrix files and the CIAO tasks mkarf and arf corr to create the point-source aperture corrected auxiliary response files. Considering no significant difference between LP observations, which were taken within 13 days, and in order to improve the statistics for spectral modeling, we created a single spectrum combining LP observations. For observation $\mathrm{C} 1$ in which the source was not detected, we used the CIAO task aprates to determine confidence bounds of the flux.

We measured the short-term variability within $0.4-3 \mathrm{keV}$ adopting the Gregory-Loredo algorithm $\frac{\sqrt{36}}{1}$ implemented by the CIAO tool

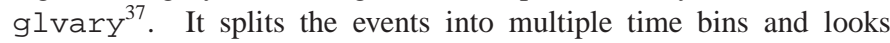
for significant deviations. The variation of the effective area with time was taken into account and was obtained by another CIAO tool dither_region. The different degrees of confidence is indicated by the parameter of "variability index", which spans values within $[0,10]$ and is larger for variability of higher confidence 37 .

Our C10 observation was intended to provide an accurate position of XJ1500+0154, utilizing the sub-arcsec resolution of Chandra near the aimpoint. We performed the source detection by applying the CIAO wavdetect wavelet-based source detection algorithm $\frac{38}{18}$ the $0.3-7 \mathrm{keV}$ image binned at single sky pixel resolution. We then carried out the absolute astrometric correction for the X-ray sources by cross-correlating them with optical sources in the Canada-FranceHawaii Telescope (CFHT) MegaPrime/MegaCam ${ }^{39} r^{\prime}$-band stacked images. We only used 19 matches that are outside the strong diffuse gas emission in NGC $5813^{40}$ and have X-ray $95 \%$ statistical positional errors $\leq 1$.'. 2 (based on Equation 12 in Kim et al ${ }^{41}$ ) and magnitude $m_{r^{\prime}}<24.0 \mathrm{AB}$ mag for astrometric correction. These 19 matches do not include $\mathrm{XJ} 1500+0154$, in order to reduce the effect of the astrometric correction on the identification of its optical counterpart. The astrometric correction method in Lin et al ${ }^{[4]}$ was used, by searching for the translation and rotation of the X-ray frame that minimize the total $\chi^{2}$ ( $\chi$ is the ratio of the X-ray-optical separation to the total positional error) for $90 \%$ (i.e., 17 , allowing 2 matches to be spurious or bad) of the matches that have the smallest $\chi$ values. The uncertainties of the translation and rotation and thus the systematic positional errors of the X-ray sources were estimated using 200 simulations. In order to calculate the statistical positional uncertainty for the source, we carried out 2000 ray-trace simulations with MARX 5.1.0 at positions near it and at the same off-axis angle. The spectrum from the thermal disk plus powerlaw fit to $\mathrm{C} 10$ was assumed.

\section{Swift observation and data analysis}

At our request, Swif $f^{[43}$ observed XJ1500+0154 in two epochs: one observation on March 28th 2014 (S1 hereafter), and seven observations between February 3rd-14th 2016 (S2-S8 hereafter, Supplementary Table 1). We analyzed the data with FTOOLS 6.18 and the calibration files released on July 31st 2015. The X-ray telescope (XRT) ${ }^{44}$ was operated in the Photon Counting mode for all observations, and we reprocessed the data with the task xrtpipeline (version 0.13.2). The spectrum was extracted, using radii of $25^{\prime \prime}$ and $2^{\prime}$ for the circular source and background regions, respectively. The source was hardly detected by the XRT in S2-S5, and we created a co-added XRT spectrum from these observations. The source was clearly detected in S6-S8, and we also created a co-added XRT spectrum from these observations. The source net count rate was higher in S6-S8 than in S2-S5 at the $3 \sigma$ confidence level.

The UV-Optical Telescope (UVOT) ${ }^{\sqrt{45}}$ in $\mathrm{S} 1$ used three standard UV filters W1 (2.3 ks), M2 (2.3 ks), and W2 (2.3 ks). In S2-S8 the UVOT used the "Filter of the Day" mode, and we combined images for different filters, resulting in total exposures of $6.3 \mathrm{ks}, 1.4 \mathrm{ks}, 3.9 \mathrm{ks}$ and $3.8 \mathrm{ks}$ for the $\mathrm{U}, \mathrm{W} 1, \mathrm{M} 2$, and W2 filters, respectively. To obtain the photometry, we used the task uvotsource with radii of $5^{\prime \prime}$ and $20^{\prime \prime}$ for the circular source and background regions, respectively.

\section{ROSAT Observations}

XJ1500+0154 was in the field of view of a ROSAT High-Resolution Imager pointed observation in 1998 for $1.9 \mathrm{ks}$ (Supplementary Table 1). XJ1500+0154 was not detected in the observation, and we extracted the source and background spectra using a circular region of radius $10^{\prime \prime}$ and a circular region of radius $50^{\prime \prime}$, respectively, in order to estimate the flux limit.

\section{CFHT MegaCam observations and data analysis}

There are seven CFHT/MegaPrime $r^{\prime}$-band images, with exposure 345 second each and taken on May 5th 2014, and eight $g^{\prime}$-band 
images, with exposure 345 second each and taken on April 26th 2014 We produced two stacked images, one for the $r^{\prime}$ band (the final seeing FWHM is $0 . .65$ ) and the other for the $g^{\prime}$ band (the final seeing FWHM is $0 .{ }^{\prime \prime} 80$ ), using MegaPipe ${ }^{46}$, and aligned their astrometry to the Sloan Digital Sky Survey (SDSS 4 . The sources detected from these two stacked images were used to compare with the SDSS photometry to detect the source optical variability and to compare with X-ray sources to align the X-ray source astrometry. We also fitted the host galaxy profile in the CFHT/MegaPrime stacked images using GALFIT ${ }^{48}$. Ten stars within $2^{\prime}$ from $\mathrm{XJ} 1500+0154$ were used to construct the point spread function of the images.

\section{X-ray spectral fits}

The X-ray activity of XJ1500+0154 should be due to accretion onto a $\mathrm{BH}$, and we fitted the X-ray spectra with several models typically used to study such an object. Given that XJ1500+0154 is most likely associated with a galaxy at $z=0.14542$, we applied this redshift to all the spectral models that we tested with the convolution model zashift in XSPEC ${ }^{49}$. All models included the Galactic absorptior 5 fixed at $N_{\mathrm{H}}=4.4 \times 10^{20} \mathrm{~cm}^{-2}$ using the TBABS model. Possible absorption intrinsic to the source was accounted for using the ztbabs model. The abundance tables from Wilms et al. ${ }^{[1]}$ were used.

Data availability statement The data that support the plots within this paper and other findings of this study are available from the corresponding author upon reasonable request.

31. Jansen, F. et al. XMM-Newton observatory. I. The spacecraft and operations. Astron. Astrophys. 365, L1-L6 (2001).

32. Strüder, L. et al. The European Photon Imaging Camera on XMMNewton: The pn-CCD camera. Astron. Astrophys. 365, L18-L26 (2001).

33. Turner, M. J. L. et al. The European Photon Imaging Camera on XMMNewton: The MOS cameras : The MOS cameras. Astron. Astrophys. 365, L27-L35 (2001). arXiv:astro-ph/0011498

34. Watson, M. G. et al. The XMM-Newton serendipitous survey. V. The Second XMM-Newton serendipitous source catalogue. Astron. Astrophys. 493, 339-373 (2009). 0807.1067

35. Bautz, M. W. et al. X-ray CCD calibration for the AXAF CCD Imaging Spectrometer. In R. B. Hoover \& A. B. Walker (ed.) Society of PhotoOptical Instrumentation Engineers (SPIE) Conference Series, vol. 3444 of Society of Photo-Optical Instrumentation Engineers (SPIE) Conference Series, 210-224 (1998).

36. Gregory, P. C. \& Loredo, T. J. A new method for the detection of a periodic signal of unknown shape and period. Astrophys. J. 398, 146-168 (1992).

37. Evans, I. N. et al. The Chandra Source Catalog. Astrophys. J. Suppl. Ser. 189, 37-82 (2010). 1005.4665

38. Freeman, P. E., Kashyap, V., Rosner, R. \& Lamb, D. Q. A WaveletBased Algorithm for the Spatial Analysis of Poisson Data. Astrophys. J. Suppl. Ser. 138, 185-218 (2002). arXiv: astro-ph/0108429

39. Boulade, O. et al. MegaCam: the new Canada-France-Hawaii Telescope wide-field imaging camera. In lye, M. \& Moorwood, A. F. M. (eds.) Instrument Design and Performance for Optical/Infrared Ground-based Telescopes, vol. 4841 of Society of Photo-Optical Instrumentation Engineers (SPIE) Conference Series, 72-81 (2003).

40. Randall, S. W. et al. Shocks and Cavities from Multiple Outbursts in the Galaxy Group NGC 5813: A Window to Active Galactic Nucleus Feedback. Astrophys. J. 726, 86 (2011). 1006.4379

41. Kim, M. et al. Chandra Multiwavelength Project X-Ray Point Source Catalog. Astrophys. J. Suppl. Ser. 169, 401-429 (2007).

42. Lin, D. et al. Discovery of the Candidate Off-nuclear Ultrasoft Hyperluminous X-ray Source 3XMM J141711.1+522541. Astrophys. J. 821, 25 (2016). 1603.00455

43. Gehrels, N. et al. The Swift Gamma-Ray Burst Mission. Astrophys. J. 611, 1005-1020 (2004).

44. Burrows, D. N. et al. The Swift X-Ray Telescope. Space Science Reviews 120, 165-195 (2005). arXiv: astro-ph/0508071

45. Roming, P. W. A. et al. The Swift Ultra-Violet/Optical Telescope. Space Science Reviews 120, 95-142 (2005). arXiv:astro-ph/0507413
46. Gwyn, S. D. J. MegaPipe: The MegaCam Image Stacking Pipeline at the Canadian Astronomical Data Centre. Publ. Astron. Soc. Pacif. 120 212-223 (2008). 0710.0370

47. Abazajian, K. N. et al. The Seventh Data Release of the Sloan Digital Sky Survey. Astrophys. J. Suppl. Ser. 182, 543-558 (2009). 0812.0649

48. Peng, C. Y., Ho, L. C., Impey, C. D. \& Rix, H.-W. Detailed Decomposition of Galaxy Images. II. Beyond Axisymmetric Models. Astron. J. 139, 2097-2129 (2010). 0912.0731

49. Arnaud, K. A. XSPEC: The First Ten Years. In Jacoby, G. H. \& Barnes, J. (eds.) Astronomical Data Analysis Software and Systems V, vol. 101 of Astronomical Society of the Pacific Conference Series, 17-+ (1996).

50. Kalberla, P. M. W. et al. The Leiden/Argentine/Bonn (LAB) Survey of Galactic HI. Final data release of the combined LDS and IAR surveys with improved stray-radiation corrections. Astron. Astrophys. 440, 775782 (2005). arXiv: astro-ph/0504140

51. Wilms, J., Allen, A. \& McCray, R. On the Absorption of X-Rays in the Interstellar Medium. Astrophys. J. 542, $914-924$ (2000). astro-ph/0008425 


\section{Supplementary Information}

\section{The X-ray source position and the host galaxy}

We obtained the position of XJ1500+0154 from $\mathrm{C} 10$ to be R.A.=15:0:52.068 and Decl.=+1:54:53.79, with the 95\% positional error of 0.18 (including both the statistical component and the systematic component from the astrometric correction procedure). This position is only 0.107 away from the center of the galaxy SDSS J150052.07+015453.8, consistent within the uncertainty (Figure 1). The number density of the galaxies that are as bright as or brighter than SDSS J150052.07+015453.8 in the $r^{\prime}$ band within $10^{\prime}$ is 0.00016 per square arcsec, implying that the chance probability for our X-ray source to be within 0 '. 18 from the center of SDSS J150052.07+015453.8 is only 0.000017. Therefore we can securely identify this galaxy as the host of our X-ray source, with the $\mathrm{X}$-ray emission most likely from the galaxy center (the projected offset $\sim 0$.' 18 or $0.5 \mathrm{kpc}$ at the redshift of $z=0.14542$ of the galaxy; see below).

The host galaxy in the CFHT/MegaPrime $r^{\prime}$ and $g^{\prime}$-band images can be fitted with a Sérsic profile, with effective radii of $0 .{ }^{\prime \prime} 48 \pm 00^{\prime \prime} 01$ $(1.23 \pm 0.03 \mathrm{kpc})$ and $0 .{ }^{\prime \prime} 41 \pm 00^{\prime \prime} 0.01(1.04 \pm 0.01 \mathrm{kpc})$, axis ratios of $0.56 \pm 0.02$ and $0.56 \pm 0.01$, and indices of $2.2 \pm 0.2$ and $3.0 \pm 0.2$, respectively. Therefore it is a small galaxy.

The SDSS took a spectrum of the galaxy on March 3rd 2011, which is shown in Supplementary Figure 1 It exhibits strong narrow emission lines at the redshift of $z=0.14542 \pm 0.00001\left(D_{L}=689 \mathrm{Mpc}\right.$, assuming a flat universe with $H_{0}=70 \mathrm{~km} \mathrm{~s}^{-1} \mathrm{Mpc}^{-1}$ and $\Omega_{\mathrm{M}}=0.3$ ). We fitted the spectrum with Penalized Pixel Fitting (pPXF) software As the stellar templates, we adopted the single-population models by Maraston and Strömbäck ${ }^{53}$ that were created based on the ELODIE library of empirical stellar spectra of solar metallicity $\sqrt{54}$ and have UV extension (below around $3950 \AA$ ) based on theoretical models $\sqrt{55}$. We chose these models because of their extended age coverage (54 ages from $3 \mathrm{Myr}$ to $12 \mathrm{Gyr})$, broad wavelength range (1000.2-6800.0 $\AA$ ), and high resolution (FWHM $\sim 0.55 \AA$ ). We interpolated the models to make them distribute uniformly in logarithm of the age from $3 \mathrm{Myr}$ to 12 Gyr with 54 grids. We fitted the SDSS spectrum over source rest-frame 3200-6800 $\AA$, considering that the spectrum at shorter wavelengths is very noisy. Fourteen Gaussian functions were also included in the fit to model the apparent gas emission lines. No additive or multiplicative polynomials were included in the fit. The spectrum was corrected for the Galactic dust reddening $\frac{\sqrt{56}}{}$ of $\mathrm{E}(\mathrm{B}-\mathrm{V})_{\mathrm{G}}=0.05 \mathrm{mag}$ before the fit. The intrinsic reddening was left as a free fitting parameter and was inferred to be $\mathrm{E}(\mathrm{B}-\mathrm{V})_{\mathrm{i}}=0.068 \mathrm{mag}$.

The pPXF fit inferred intrinsic width of the emission lines to be about $\sigma=52 \mathrm{~km} \mathrm{~s}^{-1}$. The stellar kinematics could not be constrained well, as expected considering the lack of strong absorption features. Therefore in the final fit we assumed the stellar velocity dispersion to be the same as that of the gas. The best-fit model is shown in Supplementary Figure 1 The gas emission lines are all generally consistent with a single Gaussian profile. The intrinsic reddening of the emission lines is $\mathrm{E}(\mathrm{B}-\mathrm{V})_{\mathrm{i}}=0.41 \mathrm{mag}$, assuming an intrinsic $\mathrm{H} \alpha / \mathrm{H} \beta$ ratio of 2.85. Based on more careful Gaussian fits to the star light subtracted spectrum and adopting this amount of intrinsic reddening, we obtained the extinction-corrected line ratios of $\log ([\mathrm{OIII}] \lambda 5007 / \mathrm{H} \beta)=$ $0.18 \pm 0.02$ ( $1 \sigma$ error $), \log ([\mathrm{NII}] \lambda 6583 / \mathrm{H} \alpha)=-0.63 \pm 0.02$, $\log ([\mathrm{SII}] / \mathrm{H} \alpha)=-0.79 \pm 0.04$, and $\log ([\mathrm{OI}] \lambda 6300 / \mathrm{H} \alpha)<-1.44$

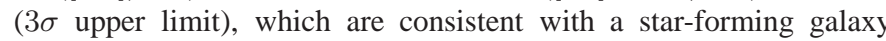
based on the BPT diagrams (Supplementary Figure 2 ${ }^{57.58}$, as indicated by the SDSS pipeline. The extinction-corrected $\mathrm{H} \alpha$ luminosity is $2.13 \pm 0.03 \times 10^{41} \mathrm{erg} \mathrm{s}^{-1}$, which implies $\sqrt{59}$ a star formation rate of $\sim 1.7 M_{\odot} / \mathrm{yr}$

However, there seem to be some small residuals around the [O III] $\lambda 5007$ line, indicating systematic blue excess. Fitting the star light subtracted spectrum around the $[\mathrm{O}$ III] $\lambda 5007$ region with two Gaussian lines, one for the strong narrow component and the other for the broad wing, we infer the wing to have $\sigma=555_{-161}^{+283} \mathrm{~km} \mathrm{~s}^{-1}$ and to be blueshifted relative to the narrow line center by $312_{-233}^{+271} \mathrm{~km} \mathrm{~s}^{-1}$. The flux of the blue wing is $1.3 \pm 0.4 \times 10^{-17} \mathrm{erg} \mathrm{s}^{-1} \mathrm{~cm}^{-2}$, corresponding to a $5.0 \sigma$ detection. Compared with the fitting residuals in other places, the residuals here are about the strongest, and thus the blue wing could be real, but we cannot completely rule out that it is due to enhanced sky noise caused by a bright sky line near [O III] $\lambda 4959$. A blue wing around [O III] $\lambda 5007$ is commonly seen in AGNs and starburst galaxies and has been interpreted as due to outflowing ionized gas $60+63$. For $\mathrm{XJ} 1500+0154$, the blue wing around [O III] $\lambda 5007$, if real, is more likely due to star-forming activity, instead of due to the X-ray outburst, as the nucleus is significantly obscured.

The mass and light (source rest-frame 3200-6800 $\AA$ ) distributions of the stellar populations with respect to the age from the pPXF fit are shown in Supplementary Figure 3 indicating the presence of some very young $(<5 \mathrm{Myr})$ populations, although the mass is dominated by old populations. The mean age weighted by light is $1.6 \mathrm{Gyr}$, and the mean age weighted by mass is $5.0 \mathrm{Gyr}$. The total stellar mass is $6.4 \times 10^{9}$ $M_{\odot}$, and the total stellar luminosity is $1.9 \times 10^{9} L_{\odot}$, suggesting a dwarf galaxy (comparable to the Large Magellanic Cloud). Using the relation between the $\mathrm{BH}$ mass and the total galaxy stellar mass ${ }^{8}$, we estimated the $\mathrm{BH}$ mass to be $1.5 \times 10^{6} M_{\odot}$ (the $1 \sigma$ uncertainty is 0.55 dex).

We do not see a clear broad component of the $\mathrm{H} \alpha$ line. Some TDEs, which would be the best interpretation for XJ1500+0154, did show broad emission lines. The well covered TDE ASASSN-14li had peak $\mathrm{H} \alpha$ luminosity of $1.1 \times 10^{41} \mathrm{erg} \mathrm{s}^{-1}$ with the corresponding FWHM of $\sim 3000 \mathrm{~km} \mathrm{~s}^{-1}$, detected in early epochs $\frac{64}{\text {. Such an }}$ emission line, however, would be difficult to detect for XJ1500+0154 if we assume the Galactic reddening of $\mathrm{E}(\mathrm{B}-\mathrm{V})_{\mathrm{G}}=0.05 \mathrm{mag}$ and intrinsic reddening of $\mathrm{E}(\mathrm{B}-\mathrm{V})_{\mathrm{i}}=0.71 \mathrm{mag}$, which is based on $\mathrm{E}(\mathrm{B}-\mathrm{V})=1.7 \times 10^{-22} N_{\mathrm{H}}$ and intrinsic column density $N_{\mathrm{H}, \mathrm{i}}=0.42 \times 10^{22} \mathrm{~cm}^{-2}$ (inferred from our X-ray spectral fits; see below). Therefore, we cannot rule out that a broad $\mathrm{H} \alpha$ line might be present in the source but is hard to detect due to strong extinction. However, it is also possible that XJ1500+0154 has intrinsically very weak optical emission (either broad emission lines or the continuum) associated with the X-ray outburst, as observed in some other TDEs during the X-ray bright phase $\mathrm{e}^{65}$

\section{The source evolution with normalized count rates}

In order to study the source behavior in a relatively modelindependent way, we calculated the count rates normalized to those expected based on the CompTT fit to the C3-C9 spectrum. Although the CompTT fit was used, other models that fit the C3-C9 spectra reasonably well (e.g., an absorbed PL) gave very similar results.

Supplementary Figure 4 shows the long-term evolution of such normalized count rates in $b=0.4-3 \mathrm{keV}, b 1=0.4-1 \mathrm{keV}$ and $b 2=1-3$ $\mathrm{keV}$. The source appears to show a prolonged outburst. It was not detected in $\mathrm{C} 1$ on April 2nd 2005, with the $0.4-3 \mathrm{keV}$ normalized count rate $R_{\mathrm{N}, \mathrm{b}}<2.3 \%$ (3 $\sigma$ upper limit, upper panel in Supplementary Figure 4. The source was detected less than 4 months later in X1 on July 23rd 2005, with $R_{\mathrm{N}, \mathrm{b}}=7.0 \pm 2.6 \%$ (90\% error). It was detected much brighter three years later, with $R_{\mathrm{N}, \mathrm{b}}=1.9 \pm 0.1$ for $\mathrm{C} 2$ on June 5th 2008 and $R_{\mathrm{N}, \mathrm{b}}=2.3 \pm 0.1$ and $2.1 \pm 0.1$ for X2 and X3, respectively, in February 2009. The source flux decayed very slowly later, with $R_{\mathrm{N}, \mathrm{b}}=1.01 \pm 0.02$ in C3-C9 in March-April 2011 and $0.35 \pm 0.04$ in $\mathrm{C} 10$ on February 23rd 2015. Similar normalized count rates were also observed in the Swift monitoring observations in 20142016, though they have poor statistics. Overall, the source count rate 
seemed to show a fast rise, by a factor of $>97$ and probably in just months, and experienced a very slow decay, by a factor of a few over a decade in time.

Comparing the $0.4-1 \mathrm{keV}$ and $1-3 \mathrm{keV}$ count rates (middle and lower panels in Supplementary Figure 4, we detected significant softening of the source in $\mathrm{C} 10$, due to a significant drop in the $1-3 \mathrm{keV}$ count rate. The normalized $0.4-1 \mathrm{keV}$ and $1-3 \mathrm{keV}$ count rates of $\mathrm{C} 10$ are $R_{\mathrm{N}, \mathrm{b} 1}=0.68 \pm 0.10$ and $R_{\mathrm{N}, \mathrm{b} 2}=0.15 \pm 0.04$, while C3-C9 has $R_{\mathrm{N}, \mathrm{b} 1}=1.01 \pm 0.03$ and $1.02 \pm 0.03$ (close to one by definition). The $R_{\mathrm{N}, \mathrm{b} 2}$ to $R_{\mathrm{N}, \mathrm{b} 1}$ ratios are $0.21 \pm 0.06$ and $1.01 \pm 0.04$ (90\% error) for $\mathrm{C} 10$ and $\mathrm{C} 3-\mathrm{C} 9$, respectively, indicating the softening of $\mathrm{C} 10$ with respect to $\mathrm{C} 3-\mathrm{C} 9$ at the $17.8 \sigma$ confidence level. In comparison, $\mathrm{C} 2, \mathrm{X} 2$, and X3 have the $R_{\mathrm{N}, \mathrm{b} 2}$ to $R_{\mathrm{N}, \mathrm{b} 1}$ ratios of $0.90 \pm 0.10,0.90 \pm 0.07$, and $0.79 \pm 0.06$, respectively, close to that of $\mathrm{C} 3-\mathrm{C} 9$. Other observations have poor statistics. S1 and S6-S8 seems to be as bright and as hard as C3-C9. S2-S5 is fainter and could be consistent with C10. X1, in the rise phase of the outburst, could have a relatively hard spectrum too.

\section{Spectral state identification for X2, X3, C2, and C3-C9}

Supplementary Table 2 gives the fit results using simple models: a PL, a MCD (diskbb in XSPEC), and their combination MCD+PL. The spectra of $\mathrm{X} 2, \mathrm{X} 3, \mathrm{C} 2$, and $\mathrm{C} 3-\mathrm{C} 9$ have similar best-fitting spectral parameters in these models, implying the same emission mechanism in these observations. The absorbed PL fits gave the photon index $\Gamma_{\mathrm{PL}} \sim$ 4-5 for $\mathrm{X} 2, \mathrm{X} 3$, and $\mathrm{C} 2$, and $\mathrm{C} 3-\mathrm{C} 9$, indicating very soft spectra and ruling out that the source was in the hard spectral state of an accreting $\mathrm{BH}$ in these observations. $\mathrm{X} 2, \mathrm{X} 3$, and $\mathrm{C} 2$ are relatively close in time (within $\sim 8$ months), and their spectra are a little softer $\left(\Gamma_{\mathrm{PL}} \sim 5\right)$ than that of $\mathrm{C} 3-\mathrm{C} 9\left(\Gamma_{\mathrm{PL}} \sim 4\right)$, taken two years later after $\mathrm{X} 2$ and $\mathrm{X} 3$. The $\mathrm{X} 2, \mathrm{X} 3$, and $\mathrm{C} 2$ spectra can be generally described with an absorbed MCD, but the fit with an absorbed MCD to C3-C9 shows systematic positive residuals at high energies $>2 \mathrm{keV}$ (Supplementary Figure 5). Adding a PL (i.e., total model MCD+PL) with $\Gamma_{\mathrm{PL}}$ fixed at a value of 2.5, typically seen in the Galactic BHBs in the thermal state, improved the fit to $\mathrm{C} 3-\mathrm{C} 9$ most significantly, but it still shows some systematic positive residuals in $2-3 \mathrm{keV}$ and negative ones above 3 $\mathrm{keV}$ (Supplementary Figure 5). In any case, the MCD+PL fits inferred a very hot disk of $k T_{\mathrm{MCD}} \sim 0.3 \mathrm{keV}$ in all spectra (the MCD+PL fits were not sensitive to the $\Gamma_{\mathrm{PL}}$ value assumed, as long as $\Gamma_{\mathrm{PL}}$ was constrained to be $\lesssim 3.5$; for higher values of $\Gamma_{\mathrm{PL}}$, the fits obtained would be dominated by a PL, instead of by a disk). We note that all fits to $\mathrm{X} 2$ tested above in fact showed some systematic residuals, whose origin will be discussed more later.

The MCD+PL fits are roughly statistically acceptable, but there are problems to associate them with the thermal state, which is characterized by a dominant thermal disk at the sub-Eddington luminosity The inferred disk temperatures of $k T_{\mathrm{MCD}} \sim 0.3 \mathrm{keV}$ are too high for such a state of an accreting SMBH. For a standard thermal disk, the maximum temperature $k T_{\mathrm{MCD}} \propto M^{-1 / 4}\left(L_{\mathrm{MCD}} / L_{\mathrm{Edd}}\right)$. The Galactic BH X-ray binaries have maximum disk temperatures normally $\lesssim 1 \mathrm{keV}$. The MCD+PL fits inferred $L_{\mathrm{X}} \sim 1-2 \times 10^{43} \mathrm{erg} \mathrm{s}^{-1}$. Therefore, for XJ1500+0154 to be at the sub-Eddington limit in the observations considered, the $\mathrm{BH}$ mass should be at least $10^{5} M_{\odot}$. Then the maximum temperature $k T_{\mathrm{MCD}}$ should be $\lesssim 0.1 \mathrm{keV}$, much lower than the values inferred. The high disk temperature might be possible if the SMBH considered is maximally spinning. To test this further, we tried to fit $\mathrm{X} 3, \mathrm{C} 2, \mathrm{C} 3-\mathrm{C} 9$ simultaneously with the more physical AGN model optxagn ${ }^{68}$ (in XSPEC). Because we are considering the thermal state, in the optxagnf model, we assumed that the gravitational energy released in the disk is emitted as a color-corrected blackbody down to a (coronal) radius $r_{\text {cor }}$, while within this radius the available energy is released in a hard PL form Comptonization component in an optically thin hot corona of temperature $100 \mathrm{keV}$. The PL index was fixed at a value of 2.5. The $\mathrm{BH}$ mass, the $\mathrm{BH}$ spin and the intrinsic column density was tied to be the same but allowed to vary. The Eddington ratios were forced to be below 1.0 (thus sub-Eddington). With all these settings, the best fit required a maximally spinning $\mathrm{BH}$ with mass $2.8 \times 10^{5} M_{\odot}$, but still a relatively high reduced $\chi^{2}$ value was obtained (1.35 for 280 degrees of freedom), and systematic fit residuals were seen clearly.

Given the above problem of fitting the spectra with the standard thermal state model MCD+PL, we tried to test the Comptonization model CompTT ${ }^{10}$ (in XSPEC). This model is commonly used to fit the

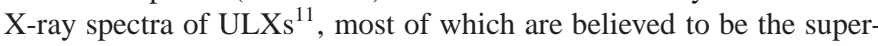
Eddington accreting stellar-mass BHs. We found that we cannot constrain all parameters well, and it seems that the fits can either assume cold seed photon solutions of $k T_{0} \lesssim 0.1 \mathrm{keV}$ or hot seed photon solutions of $k T_{0} \sim 0.1-0.2 \mathrm{keV}$, corresponding to two local/global minima in $\chi^{2}$, which differed by $<6$. Cold seed photon solutions were associated with higher column densities $\left(N_{\mathrm{H}} \sim 0.4-0.5 \times 10^{22} \mathrm{~cm}^{-2}\right)$ than hot seed photon solutions $\left(N_{\mathrm{H}} \sim 0.1-0.2 \times 10^{22} \mathrm{~cm}^{-2}\right)$. In the case of ULXs, the seed photon temperatures were often inferred ${ }^{11}$ to be $\lesssim 0.3$ $\mathrm{keV}$. If our spectra have the same emission mechanism as most ULXs and the seed photons are from a thermal disk, we would expect the seed photons in our spectra to be very cool, $<0.1 \mathrm{keV}$. Therefore we will focus on the cold seed photon solutions. Still, we found that in order to obtain well constrained corona temperatures and optical depths for better comparison between observations, it is desirable to fix the intrinsic column density $N_{\mathrm{H}, \mathrm{i}}$ and the seed photon temperature $k T_{0}$. From the simultaneous fits to $\mathrm{X} 3, \mathrm{C} 2$, and $\mathrm{C} 3-\mathrm{C} 9$ spectra with these parameters tied to be the same (X2 was not included, again due to presence of fit residuals), we inferred $N_{\mathrm{H}, \mathrm{i}}=0.42 \pm 0.06 \times 10^{22} \mathrm{~cm}^{-2}$ and $k T_{0}=0.04 \pm 0.04 \mathrm{keV}$. Therefore in the final fits with CompTT, we fixed $N_{\mathrm{H}}$ at $0.42 \times 10^{22} \mathrm{~cm}^{-2}$ and $k T_{0}$ at $0.04 \mathrm{keV}$. The fit results are given in Table 1. The disk geometry was assumed (the fits of similar quality can also be obtained assuming a spherical geometry, which would infer similar corona temperatures but higher (by a factor of $\sim 2$ ) values of the optical depth).

From Table 1, we see that the CompTT fits suggest a cool $\left(k T_{\mathrm{e}} \sim\right.$ $0.35-1.3 \mathrm{keV})$ optically thick $(\tau \sim 4-11)$ corona for X2, X3, C2 and C3-C9. C3-C9 might have a little hotter corona and a lower optical depth than earlier observations X2, X3, and C2. To test the significance of the presence of a cool optically thick corona in these spectra, we still tried to fit $\mathrm{X} 3, \mathrm{C} 2$, and $\mathrm{C} 3-\mathrm{C} 9$ simultaneously, with the seed photon temperatures all fixed at $0.04 \mathrm{keV}$, the corona temperatures all fixed at $20.0 \mathrm{keV}$, and the column densities allowed to vary freely but all tied to be the same. Then we obtained a total $\chi^{2}$ value of 257.6 for 282 degrees of freedom. In comparison, the total $\chi^{2}$ value is 242.3 for 3 fewer degrees of freedom if the corona temperature parameters are all allowed to vary freely. Based on the $F$-test, this implies a $3.4 \sigma$ improvement of the fits with a cool optically thick corona over those with a hot optically thin one, under the assumption of cool seed photons. Therefore there is evidence that $\mathrm{XJ} 1500+0154$ was in a super-Eddington accretion state in $\mathrm{X} 2, \mathrm{X} 3, \mathrm{C} 2$, and $\mathrm{C} 3-\mathrm{C} 9$.

We note that all the spectral models tested above on $\mathrm{X} 2$ all showed systematic fit residuals, as shown in Supplementary Figure 5 $\mathrm{X} 2$ was taken only six days before X3, and they have very similar instrument configurations and source off-axis angles. The source spectral shape and count rates are also very similar in these two observations, which seems to suggest that the residuals in X2 could be due to some calibration uncertainty, or due to presence of a warm absorber in X2. Starting with the continuum model CompTT with $N_{\mathrm{H}, \mathrm{i}}$ fixed at $0.42 \times 10^{22} \mathrm{~cm}^{-2}$ and $k T_{0}$ fixed at $0.04 \mathrm{keV}$, we found that the residuals can be significantly reduced by adding an edge of threshold energy $E_{\text {edge }}=0.63 \pm 0.03 \mathrm{keV}$ and optical depth $\tau=0.88 \pm 0.30$ (corresponding to a $5 \sigma$ improvement) and another 
edge of $E_{\text {edge }}=1.33 \pm 0.07 \mathrm{keV}$ and $\tau=0.37 \pm 0.21$ (corresponding to a $3 \sigma$ improvement). Replacing these edges with the more physical model zxipcf, we obtained two possible fits. One inferred an absorber of column density $N_{\mathrm{H}}=0.4 \times 10^{22} \mathrm{~cm}^{-2}$, ionizing parameter $\log (\xi)=-0.55$ (i.e., nearly neutral), and zero speed (i.e., the absorber is static relative to the source), and required the unabsorbed luminosity to be a factor of 6.7 higher than that of X3. The other fit inferred an absorber of $N_{\mathrm{H}}=3.5 \times 10^{22} \mathrm{~cm}^{-2}, \log (\xi)=2.7$ and redshifted speed of $29 \%$ the speed of the light (thus an inflow), with the unabsorbed source luminosity similar to that of X3. Both fits accounted for most of the residuals around $0.6 \mathrm{keV}$ and are clearly challenging to understand, with the former requiring a large change in luminosity in a short time and the latter requiring a fast warm inflow. Future observations would be helpful to determine whether the residuals are real

\section{Spectral state identification for $\mathbf{C 1 0}$}

We also have a relatively good spectrum from $\mathrm{C} 10$, which appears to be much softer than earlier observations, as shown above based on the normalized count rates in different energy bands. The absorbed PL fit inferred a very high photon index of $\Gamma_{\mathrm{PL}}=8.0 \pm 1.2$ for $\mathrm{C} 10$, compared with $\Gamma_{\mathrm{PL}} \sim 4-5$ for X2, X3, C2 and C3-C9. The MCD+PL fit with $\Gamma_{\mathrm{PL}}$ fixed at a value 2.5 also indicates that the spectrum in $\mathrm{C} 10$ is dominated by a disk with a much lower temperature in $\mathrm{C} 10$ than in $\mathrm{X} 2, \mathrm{X} 3, \mathrm{C} 2$ and C3-C9 (0.13 keV versus $\sim 0.3 \mathrm{keV})$.

One explanation for the spectral softening in $\mathrm{C} 10$ is that the source showed a transition from the super-Eddington accretion state in earlier observations to the thermal state in $\mathrm{C} 10$. Then the MCD+PL fit would be a reasonable model. Table 1 lists such a fit with $N_{\mathrm{H}, \mathrm{i}}$ fixed at $4.2 \times$ $10^{21} \mathrm{~cm}^{-2}$, as obtained from the simultaneous CompTT fits to X3, C2, and C3-C9. This model inferred $L_{\mathrm{X}}$ in C10 only lower than that in C3-C9 by $15 \%$. We note that in the second ULX in M31, similar state transition also occurred with luminosity changing by $<20 \%$.

Alternatively, the spectral softening in $\mathrm{C} 10$ could be due to a transient warm absorber in C10 that obscured the high-energy flux. To test this scenario, we added a warm absorber to the CompTT fit to C3-C9. The warm absorber was modelled with the zxipcf (in XSPEC) model. The CompTT parameters were fixed at the values obtained from the fit to $\mathrm{C} 3-\mathrm{C} 9$. The covering fraction was found to be consistent with 1.0 (the $90 \%$ lower confidence bound is 0.91 ), and we thus fixed it at the value of 1.0. Then we inferred the warm absorber to have $N_{\mathrm{H}}=64 \times 10^{22} \mathrm{~cm}^{-2}, \log (\xi)=2.8$ and blueshifted velocity of $0.36 \mathrm{c}$ (Table 1). This fit has almost the same $C$ statistic as the $\mathrm{MCD}+\mathrm{PL}$ fit. If the normalization of the CompTT model is allowed to vary, considering that the source might become fainter, we found that the unabsorbed luminosity in $\mathrm{C} 10$ should be $>88 \%$ (90\% lower bound, the upper bound cannot be constrained, as there is degeneracy between the source flux and the column density of the absorber) of that of C3-C9. A highly ionized sub-relativistic outflowing absorber is expected in the super-Eddington accretion phase of TDEs 1 and had been inferred in a few cases $\sqrt{69,70}$.

\section{Spectral state identification for Swift observations}

The Swift observations are all too faint to carry out detailed spectral fits. In S1, S2-S5, and S6-S8, we collected 18.3, 6.0 and 18.8 net counts within $0.3-10 \mathrm{keV}$ in exposures of $8.7 \mathrm{ks}, 9.0 \mathrm{ks}$, and $6.7 \mathrm{ks}$, respectively. Based on the normalized count rates shown in Supplementary Figure 4 the source spectra in S1 and S6-S8 are more consistent with that in $\mathrm{C} 3-\mathrm{C} 9$ than with that in $\mathrm{C} 10$. The normalized 1-3 keV count rates in $\mathrm{S} 1$ and S6-S8 are both consistent with that in $\mathrm{C} 3-\mathrm{C} 9$ to within $1 \sigma$ error but both different from that in $\mathrm{C} 10$ at the $2.5 \sigma$ confidence level. Therefore the source might be still in the super-Eddington accretion state in S1 and S6-S8. In contrast, the low $0.4-3 \mathrm{keV}$ normalized count rate in S2-S5 makes it more consistent with $\mathrm{C} 10$ (within $1 \sigma$ ) than $\mathrm{C} 3-\mathrm{C} 9$, and the source could be in the thermal state or subject to transient absorption again in this observation. Given the low statistics of the Swift observations, however, we cannot completely rule out that the source had similar $\mathrm{X}$-ray spectra in S2-S8 as in C10, which could suggest that the source had been completely settled to the thermal state since C10. Deeper observations of the source in the future are needed to firmly determine the duration of the super-Eddington accretion phase.

\section{Spectral state identification for X1}

The spectrum of X1 has low quality too. Fitting it with a PL gave $\Gamma_{\mathrm{PL}}=2.7_{-0.7}^{+2.3}$. Thus it could be a little harder than other observations. We tried to fit it with the MCD+PL model with $N_{\mathrm{H}, \mathrm{i}}$ fixed at $4.2 \times 10^{21}$ $\mathrm{cm}^{-2}$ and the PL photon index fixed at the value of 2.5 . We obtained a fit with a cool disk of $k T_{\mathrm{MCD}}=0.10_{-0.04}^{+0.06} \mathrm{keV}$ and a PL contributing about $25 \%$ of the rest-frame $0.34-11.5 \mathrm{keV}$ luminosity.

The identification of the spectral state for X1 is clearly non-trivial due to its low statistics, but it is consistent with a transitional state, as expected considering that it was at the beginning of the outburst.

\section{Fitting the long-term $\mathrm{X}$-ray luminosity curve}

The evolution of the X-ray luminosity in TDEs depends on many factors and can involve physics that is still poorly understood, especially in the super-Eddington accretion regime. We explored the models that could describe the data assuming full disruption of a star by a $10^{6} M_{\odot}$ black hole. The accretion rate of the mass was assumed to be slowed relative to the fallback rate by the viscous timescale $\tau_{\text {visc }}$, according to the equation

$$
\dot{M}_{\mathrm{d}}(t)=\frac{1}{\tau_{\mathrm{visc}}}\left(e^{-t / \tau_{\mathrm{visc}}} \int_{0}^{t} e^{t^{\prime} / \tau_{\mathrm{visc}}} \dot{M}_{\mathrm{fb}}\left(t^{\prime}\right) d t^{\prime}\right),
$$

where $\dot{M}_{\mathrm{d}}$ is the mass accretion rate and $\dot{M}_{\mathrm{fb}}$ is the mass fallback rate $e^{71}$. The radiative efficiency was assumed to be 0.1 when the accretion rate $\dot{M}_{\mathrm{d}}$ is below the 0.5 isotropic Eddington limit $\dot{M}_{\mathrm{Edd}}$. At higher accretion rates, the inner disk could begin to reach the local Eddington limi ${ }^{72}$, and we assumed the luminosity to scale logarithmically, in the form $\frac{13}{13}$ of $1.0+\log \left(\dot{M}_{\mathrm{d}} / 0.5 \dot{M}_{\text {Edd }}\right)$. Based on the X-ray spectral fits, we assumed that $25 \%$ of the light was emitted in the X-ray band (the source rest-frame $0.34-11.5 \mathrm{keV}$ ).

Supplementary Figure 6 shows the expected light curves for several models of different masses of the disrupted star and different viscous timescales. For comparison, models incorporating the super-Eddington accretion effects are shown as thick black lines, while those not are shown as thin gray lines (representing the evolution of the fallback rate in case of prompt circularization).

We find that there is degeneracy between the stellar mass and $\tau_{\text {visc }}$. Our data can be described with the full disruption of a $2 M_{\odot}$ star with $\tau_{\text {visc }}=3 \mathrm{yr}$ (Figure 2 ) or a $10 M_{\odot}$ star with $\tau_{\text {visc }}=0$ (Supplementary Figure 6). The degeneracy can be broken with more future monitorings of the source, as the luminosity is expected to decay faster for disruption of a lower mass star. Assuming a higher efficiency in either the Eddington excess or in the mass-to-light conversion would adjust the favored stellar masses downward, with a maximally-spinning black hole driving the favored masses to values of 0.5 and $2.5 M_{\odot}$, respectively.

While the case with a larger star describes our data just as well as the case with a star of nearly a solar mass, the steep stellar mass function strongly favors the disruptions of lower-mass stars, even in cases

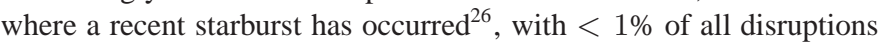
coming from stars with $M \geq 10 M_{\odot}$. Additionally, we expect that disruptions of stars around $10^{6} M_{\odot}$ black holes will be slowed by viscous effects in the majority of disruptions, with two-thirds of disruptions 
having viscous timescales longer ${ }^{\sqrt{19}}$ than a year for $M_{\mathrm{h}}<10^{6} M_{\odot}$. Because of these expectations, we argue that XJ1500+0154 most likely originated from the disruption of a nearly solar-mass star.

A single strong disruption and prompt accretion of an evolved star, either ascending the red giant branch or on the horizontal branch, can produce a prolonged TDE with a fast rise and a prolonged superEddington accretion phase ${ }^{73}$, as seen in $\mathrm{XJ} 1500+0154$. However, TDEs of evolved stars are expected to be very rare for a black hole of mass $10^{6} M_{\odot}$, accounting for $\sim 1 \%$ of the total TDEs ${ }^{26}$, and they should be dominated by repeated, partial disruptior ${ }^{74}$. Single strong disruptions of evolved stars should be extremely rare, and we do not expect to detect any in the limited volumn of space searched (see below).

\section{ROSAT Observations}

XJ1500+0154 was not detected in the ROSAT All-Sky Survey in 1990. The survey had a detection limi ${ }^{75}$ of the $0.1-2.4 \mathrm{keV}$ flux of $5 \times 10^{-13} \mathrm{erg} \mathrm{s}^{-1} \mathrm{~cm}^{-2}$, which is a factor of 3 higher than the peak absorbed 0.1-2.4 keV (observer-frame) flux of XJ1500+0154 $\left(\sim 1.7 \times 10^{-13} \mathrm{erg} \mathrm{s}^{-1} \mathrm{~cm}^{-2}\right.$ in X2 and X3). The source was not detected either in the ROSAT/HRI pointed observation in 1998. The $3 \sigma$ upper limit of the source net count rate is 0.0038 counts s ${ }^{-1}$, which is about 50\% lower than that expected from X2 and X3. Therefore, although the source was not detected in ROSAT observations, they are not deep enough for us to rule out the emission level seen in the current outburst.

\section{Short-term X-ray variability}

Supplementary Figure 7 shows the light curves for all bright observations (X2-X3 and $\mathrm{C} 2-\mathrm{C} 10)$. We obtained $\chi^{2}$ probability of constancy of 0.13 and $2.9 \times 10^{-4}$ from the $0.3-3 \mathrm{keV}$ pn light curves binned at $500 \mathrm{~s}$ in the $\mathrm{X} 2$ and $\mathrm{X} 3$ observations, respectively. Therefore, no short-term variability was detected from X2. X3 might show some variability, with a fast (within $2 \mathrm{ks}$ ) drop to almost zero count rate at around $20 \mathrm{ks}$ into the observation. The average count rates of X2 and X3, which are six days apart, are, however, consistent with each other within $2 \sigma$ (Supplementary Table 1). We detected no short-term variability in the Chandra observations C2-C10, all with a Gregory-Loredo variability index of 0 . We note that the lower count rates in the Chandra observations (a factor of $\gtrsim 2$ lower than the XMM-Newton/pn in X2 and X3) could make it hard to detect the fast drop as is possibly present in X3. The observations C3-C9, taken within a 13 day period, have count rates consistent with each other within $2 \sigma$ (Supplementary Table 1 ).

\section{UV and optical long-term variability}

The SDSS photometry of the host galaxy on May 23rd 2001 is $u^{\prime}=$ $21.71 \pm 0.35 \mathrm{AB}$ mag, $g^{\prime}=20.96 \pm 0.07 \mathrm{AB}$ mag, $r^{\prime}=20.34 \pm 0.06$ $\mathrm{AB}$ mag, $i^{\prime}=19.97 \pm 0.07 \mathrm{AB}$ mag, and $z^{\prime}=19.81 \pm 0.27 \mathrm{AB}$ mag (Petrosian magnitudes). The corresponding CFHT/MegaPrime $r^{\prime}$ and $g^{\prime}$ photometry can be obtained through the relation $r_{\text {Mega }}^{\prime}=$ $r_{\text {SDSS }}^{\prime}-0.024\left(g_{\text {SDSS }}^{\prime}-r_{\text {SDSS }}^{\prime}\right)=20.32 \pm 0.06 \mathrm{AB}$ mag, and $g_{\text {Mega }}^{\prime}=g_{\text {SDSS }}^{\prime}-0.153\left(g_{\text {SDSS }}^{\prime}-r_{\text {SDSS }}^{\prime}\right)=20.87 \pm 0.07 \mathrm{AB}$ mag. In comparison, we measured $r^{\prime}$ Mega $=20.25 \pm 0.01 \mathrm{AB}$ mag and $g_{\text {Mega }}^{\prime}=20.92 \pm 0.01 \mathrm{AB}$ mag from the CFHT/MegaPrime stacked images in 2014. Therefore the photometry in each filter was consistent with each other in these two epochs to within the $1 \sigma$ uncertainty. The $3 \sigma$ upper limit in the $r^{\prime}$ band in 2014 due to the flare is about $\lambda L_{\lambda}<1.3 \times 10^{42} \mathrm{erg} \mathrm{s}^{-1}$ or $<3.5 \times 10^{8} L \odot$. If we adopt the reddening relation $\mathrm{E}(\mathrm{B}-\mathrm{V})=1.7 \times 10^{-22} N_{\mathrm{H}}$ and use the column density of $N_{\mathrm{H}, \mathrm{i}}=4.2 \times 10^{21} \mathrm{~cm}^{-2}$ inferred from the X-ray spectral fits, the above limit after extinction correction would be an order of magnitude larger (i.e., $<3.5 \times 10^{9} L_{\odot}$ ). The three ASASSN TDEs have $\lambda L_{\lambda}$ in the range between $10^{9}$ and $10^{10} L_{\odot}$ at $\lambda=5400 \AA$ (the source restframe central wavelength of the $r^{\prime}$ band for XJ1500+0154) at the very early epoch of the events ${ }^{76}$. Therefore we cannot rule out a similar level of optical emission as seen in the ASASSN TDEs in our event.

There is a faint UV source from the Swift observation at the position of the SDSS galaxy. We obtained the W1, M2, and W2 magnitudes of $23.1 \pm 0.5 \mathrm{AB}$ mag, $23.3 \pm 0.5 \mathrm{AB}$ mag, and $23.8 \pm 0.7 \mathrm{AB}$ mag, respectively, from $\mathrm{S} 1$, and $23.5 \pm 1.4 \mathrm{AB}$ mag, $22.8 \pm 0.3 \mathrm{AB}$ mag, and $22.9 \pm 0.3 \mathrm{AB}$ mag, respectively, from $\mathrm{S} 2-\mathrm{S} 8$. The relatively large errors are due to the scattered light background from a nearby bright star. The UV source was also detected by GALEX on June 3rd, 2007, with the NUV and FUV magnitudes of $22.9 \pm 0.3 \mathrm{AB}$ mag and $23.8 \pm 0.3$ $\mathrm{AB}$ mag (the NUV filter has an effective wavelength similar to that of the Swift M2 filter, i.e., 2230 ̊), The blue UV emission is consistent with each other in these epochs and is consistent with that expected from emission from the young stellar populations based on the pPXF fit to the SDSS spectrum of the galaxy. The UV emission from the three ASASSN TDEs in the early epoch ${ }^{76}$ would correspond to a W2 magnitude of $>27 \mathrm{AB}$ mag for XJ1500+0154 if we also applied the extinction based on $\mathrm{E}(\mathrm{B}-\mathrm{V})=1.7 \times 10^{-22} N_{\mathrm{H}}$ and used the column density of $N_{\mathrm{H}, \mathrm{i}}=4.2 \times 10^{21} \mathrm{~cm}^{-2}$. The $\mathrm{U}$ band magnitude in $\mathrm{S} 2-\mathrm{S} 8(22.1 \pm 0.3 \mathrm{AB} \mathrm{mag})$ is also consistent with the stellar emission.

\section{Arguments against the AGN explanation}

The coincidence with an optical galactic center, with chance coincidence probability of only 0.000017 , led us to conclude that $\mathrm{XJ} 1500+0154$ is due to nuclear activity in SDSS J150052.07+015453.8. The significant absorption column density other than the Galactic value required in the X-ray spectral fits reassures this association. Then we are left with two possible interpretations of the source: a persistent AGN or a TDE.

As we have shown, we see no sign of persistent nuclear activity in the optical spectrum, which shows no clear broad emission lines but narrow ones, with the ratios fully consistent with those expected for starburst galaxies. For AGNs, there is a strong correlation between the persistent hard X-ray luminosity and the extinction-corrected [O III] $\lambda 5007$ luminosity $L_{\mathrm{OIII}}^{\mathrm{c}}$. XJ1500+0154 has $L_{\mathrm{OIII}}^{\mathrm{c}}=2.9 \pm 0.1 \times 10^{41}$ $\mathrm{erg} \mathrm{s}^{-1}$ if we assume $\mathrm{E}(\mathrm{B}-\mathrm{V})_{\mathrm{G}}=0.05$ mag and $\mathrm{E}(\mathrm{B}-\mathrm{V})_{\mathrm{i}}=0.71$ mag, which is again based on $\mathrm{E}(\mathrm{B}-\mathrm{V})=1.7 \times 10^{-22} N_{\mathrm{H}}$ and intrinsic column density $N_{\mathrm{H}, \mathrm{i}}=0.42 \times 10^{22} \mathrm{~cm}^{-2}$ inferred from our X-ray spectral fits. The $2-10 \mathrm{keV}$ luminosity corresponding to this $L_{\mathrm{OIII}}^{\mathrm{c}}$ is ${ }^{77}$ $3.6 \times 10^{42} \mathrm{erg} \mathrm{s}^{-1}$ (the dispersion is $0.63 \mathrm{dex}$ ). The corresponding 0.34-2.0 keV unabsorbed luminosity assuming a PL of photon index 2.0 is $3.9 \times 10^{42} \mathrm{erg} \mathrm{s}^{-1}$, which is about an order of magnitude lower than the flux seen in the outburst. Given that the line ratios are fully consistent with the star-forming activity, the [O III] $\lambda 5007$ flux should be dominated by the star-forming activity, instead of that of the nucleus. Then the persistent unabsorbed $0.34-2 \mathrm{keV}$ luminosity implied by the optical spectrum would be much lower than observed in the ourburst, arguing against a persistent AGN explanation for XJ1500+0154.

We can also compare XJ1500+0154 with 753 spectroscopically identified AGNs in Lin et al. ${ }^{[7]}$. These AGNs were included in that study because they had multiple observations, with at least one detection with $\mathrm{S} / \mathrm{N}>20$, in the $2 \mathrm{XMM}-\mathrm{DR} 3$ catalog. Supplementary Figure 8 shows an X-ray color-color diagram, adapted from Figure 2 in Lin et al., by using the $3 \mathrm{XMM}-\mathrm{DR} 5$ catalog. The X-ray colors HR2 and HR3 are defined as $(H-S) /(H+S)$, with $S$ and $H$ being the MOS1-medium-filter equivalent $0.5-1 \mathrm{keV}$ and $1-2 \mathrm{keV}$ counts rates for HR2 and 1-2 keV and 2-4.5 keV count rates for HR3, respectively. The MOS1-medium-filter equivalent count rates are those expected for an on-axis MOS1 observation using a "Medium" optical filter ${ }^{78}$. In terms of HR3, which characterizes the spectral shape within $1-4.5 \mathrm{keV}$, $\mathrm{XJ} 1500+0154$ is significantly softer (HR3 in the range between -0.78 
and -0.86 for all bright observations $\mathrm{C} 2, \mathrm{C} 3-\mathrm{C} 9, \mathrm{C} 10, \mathrm{X} 2$ and $\mathrm{X} 3$ ) than AGNs (HR3 $\gtrsim-0.70$ ).

The long-term variability of $\mathrm{XJ} 1500+0154$, a factor of $>97(3 \sigma$ lower limit), is also extreme, compared with AGNs. Only one out of 753 AGNs in Lin et al. varied by a factor of $>97$ based on the 3XMMDR5 catalog. The large variability in AGNs has been normally ascribed to all kinds of absorbers ${ }^{79}$. In order to explain the non-detection of $\mathrm{XJ} 1500+0154$ in $\mathrm{C} 1$ and the low count rate in $\mathrm{X} 1$, it would require a neutral absorber of $N_{\mathrm{H}}>6.8 \times 10^{22} \mathrm{~cm}^{-2}$ and $N_{\mathrm{H}}=2.5 \times 10^{22}$ $\mathrm{cm}^{-2}$ fully covering a $\mathrm{X} 2$ spectrum in $\mathrm{C} 1$ and $\mathrm{X} 1$, respectively. The problem with this explanation is that it would imply no detection of the source below $1 \mathrm{keV}$ in $\mathrm{X} 1$, while we had detected the source in $0.4-1$ $\mathrm{keV}$ at the $3.2 \sigma$ confidence level in this observation, unless the absorber has a complex structure. Besides, this explanation has to require highly variable absorption of an AGN with unusually soft X-ray spectra.

A very small number of high-amplitude very soft X-ray flares were detected from galactic nuclei that might have low persistent luminosities as inferred from the optical emission lines ${ }^{80-87}$. It is under debate on whether these flares are due to, e.g., disk instability in AGNs, or due to TDEs. They might even represent a mixed class, as they showed a variety of spectral shape and temporary evolution. XJ1500+0154 is different from them in several aspects (e.g., generally higher characteristic temperatures and dramatic spectral softening in XJ1500+0154). Therefore it is not clear whether XJ1500+0154 has the same origin as the other flares.

There are several AGNs known to show significant spectral changes, between type 1 and type 2 in optica $\frac{88.90}{\text { and/or between }}$ Compton-thin and reflection-dominated states in X-rays $\frac{9192}{\text { ? There }}$ are various explanations for them, including variable absorption and changes in the accretion rate (due to, e.g., a TDE). Supplementary Figure 8 in fact includes some such changing-look AGNs, e.g., NGC 1365 and 1H 0707-495. These AGNs change between two AGN standard spectral states with hard X-ray spectra. XJ1500+0154 is different from them in that it changed between two states unseen in standard AGNs, with very soft X-ray spectra.

Dwarf starburst galaxies hosting luminous AGNs are also extremely rare in the local Universe. Only a small number of dwarf galaxies are known to show active nucle 93.96 . Among them, only Henize $2-10$ is in a starburst galaxy, with extremely low luminosity in the nucleus 95

The column density inferred from the X-ray spectral fits is optically thin to Thomson electron scattering 97 . Then the wind from the central source can sweep up the gas into a shell and push it outwards at a velocity ${ }^{98}$ of $v_{s}=\left(f_{w} L 8 \pi^{2} G / f_{\text {gas }} \sigma^{2}\right)^{1 / 3}=2700\left(f_{\mathrm{w}} / f_{\text {gas }}\right)^{1 / 3}$ $\mathrm{km} \mathrm{s}^{-1}$, where $f_{\text {gas }}$ is the mass fraction of the gas, $\sigma=52 \mathrm{~km} \mathrm{~s}^{-1}$ is the velocity dispersion, $G$ is gravitational constant, $L$ is the source bolometric radiation luminosity, $\sim 10^{44} \mathrm{erg} \mathrm{s}^{-1}$, and $f_{\mathrm{w}} L$ is the wind energy. Given the super-Eddington accretion nature of the source, we expect $f_{\mathrm{w}} / f_{\text {gas }}$ to be $>1.0$. Therefore $v_{s}>2700 \mathrm{~km} \mathrm{~s}^{-1}$, and gas within $1 \mathrm{kpc}$ (the effective radius of the host galaxy) can be swept out in $<0.4 \mathrm{Myr}$, which is very short. Therefore the persistent luminous AGN explanation is hard to be reconciled with the star-forming activity of the host galaxy.

We note that we do not have deep radio observations of XJ1500+0154 yet and that it was not detected in the radio surveys NVSS or FIRST. The low sensitivity of these surveys did not allow us to rule out a persistent AGN based on the radio upper limit.

\section{Comparison with other candidate TDEs}

There are about 30 TDEs discovered thus far that are bright in Xrays 99100 . Most of them had peak X-ray luminosity $<10^{44} \mathrm{ergs} \mathrm{s}^{-1}$, fast decay by about one order of magnitude in a year, and super-soft $X$ -

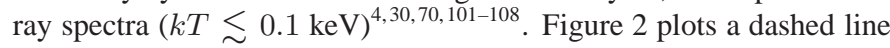

showing a representative light curve for such super-soft TDEs. Our event, evolving on a timescale at least two orders of magnitude longer, having much harder X-ray spectra, and exhibiting dramatic spectral softening, is different from them.

Recently, three TDEs with hard X-ray spectra (photon index around 2.0) and apparent peak luminosity highly super-Eddington were also found 109:113. They are also short-lived, decreasing by more than two orders of magnitude in the first year. Our event is distinguished from them, with much softer X-ray spectra, long duration, and dramatic X-ray spectral change.

\section{Event rate}

We roughly estimated the rate of TDEs with a super-Eddington accretion phase, characterized by quasi-soft X-ray spectra. XJ1500+0154 was discovered through the search over the detections in the 3XMMDR5 catalog that have $\mathrm{S} / \mathrm{N}>20$. We selected out sources that showed large variability and/or soft spectra (e.g., HR3 $<-0.7$ ). Based on observations outside the Galactic plane (the galactic latitude $|b|>20^{\circ}$ ) and assuming that events like XJ1500+0154 have a mean luminosity three times lower than that in X2 but have similar quasi-soft X-ray spectra and last for 10 years, we estimated that we should be able to detect about $1.8 \times 10^{8} \mathrm{rn}$ events, where $r$ is the event rate per galaxy per year and $n$ is the galaxy density per $\mathrm{Mpc}^{3}$. We assumed ${ }^{25} n=1.4 \times 10^{-2}$ $\mathrm{Mpc}^{-3}$. We only discovered one with quasi-soft X-ray spectra, indicating $r \sim 4 \times 10^{-7} \mathrm{gal}^{-1} \mathrm{yr}^{-1}$. This is a conservative estimate because our search was still preliminary and the absorption effect was not taken into account. The real rate could be a factor of a few larger.

The above event rate calculation depends on the assumption of the duration of the super-Eddington accretion phase in the events. Assuming shorter durations would proportionally infer higher rates. Therefore we cannot rule out a much higher rate for all super-Eddington accreting TDEs as their super-Eddington accretion phase could be generally shorter than that of our event. Although super-Eddington accretion in TDEs is probably rarer than previously thought, due to slow circularization effects, a significant fraction of TDEs are still expected to show a super-Eddington accretion phase ${ }^{\sqrt{19}}$. Guided by the discovery of XJ1500+0154, we tried to search for TDEs with quasi-soft X-ray spectra (thus suggesting a super-Eddington accretion phase) from newly public XMM-Newton observations and have discovered two candidates (Lin et al. 2017, in preparation). They have peak X-ray luminosity of $\sim 10^{44} \mathrm{erg} \mathrm{s}^{-1}$. One of them is clearly in an outburst, with a rising time less than 10 months, and the host galaxy showed no optical emission lines, ruling out the presence of a persistently bright nucleus. The other one is consistent to be in an outburst too, with two X-ray observations showing the flux decay by a factor of $\sim 6$ in 1.5 years, and the host is a star-forming galaxy (or at the end phase of a starburst phase, given the strong Balmer absorption). The durations of these two events still need to be constrained with future observations. Discovery of additional quasi-soft X-ray objects in bright outbursts and in hosts showing no sign of persistent nuclear activity in the optical spectra strongly support that they are super-Eddington accreting TDEs and that such events might not be so rare. 
Supplementary Table 1 | The X-ray Observation Log. Columns: (1) the observation ID with our designation given in parentheses, (2) the observation start date, (3) the detector, (4) the off-axis angle, (5) the exposures of data used in final analysis, (6) radius of the source extraction region, (7) the net count rate in the source extraction region (0.3-3 keV for XMM-Newton and 0.4-3 keV for Chandra and Swift observations), with $1 \sigma$ error (but for observations rh601125n00, C1 and S2-S5, the $3 \sigma$ confidence bounds are given).

\begin{tabular}{|c|c|c|c|c|c|c|}
\hline $\begin{array}{l}\text { Obs. ID } \\
\text { (1) }\end{array}$ & $\begin{array}{l}\text { Date } \\
(2)\end{array}$ & $\begin{array}{l}\text { Detector } \\
\text { (3) }\end{array}$ & $\begin{array}{c}\text { OAA } \\
(4)\end{array}$ & $\begin{array}{c}T(\mathrm{ks}) \\
(5)\end{array}$ & $\begin{array}{c}r_{\mathrm{src}} \\
(6)\end{array}$ & $\begin{array}{c}\text { Count rate }\left(10^{-3} \text { counts } \mathrm{s}^{-1}\right) \\
(7)\end{array}$ \\
\hline \multicolumn{7}{|l|}{ rh601125n00 } \\
\hline \multicolumn{7}{|l|}{ XMM-Newton: } \\
\hline $0302460101(\mathrm{X} 1)$ & $2005-07-23$ & $\mathrm{pn} / \mathrm{M} 1 / \mathrm{M} 2$ & 13.5 & $22 / 33 / 33$ & $20^{\prime \prime} / 20^{\prime \prime} / 20^{\prime}$ & $1.45 \pm 0.40 / 0.74 \pm 0.20 / 0.21 \pm 0.15$ \\
\hline $0554680201(\mathrm{X} 2)$ & $2009-02-12$ & $\mathrm{pn} / \mathrm{M} 2$ & $12^{\prime} \cdot 5$ & $43 / 64$ & $20^{\prime \prime} / 20^{\prime \prime}$ & $37.4 \pm 1.0 / 11.1 \pm 0.4$ \\
\hline $0554680301(X 3)$ & $2009-02-18$ & $\mathrm{pn} / \mathrm{M} 2$ & $12^{\prime} \cdot 5$ & $42 / 64$ & $20^{\prime \prime} / 20^{\prime \prime}$ & $35.6 \pm 0.9 / 9.7 \pm 0.4$ \\
\hline \multicolumn{7}{|l|}{ Chandra: } \\
\hline $5907(\mathrm{C} 1)$ & $2005-04-02$ & ACIS-S1 & $12^{\prime} \cdot 4$ & 48 & $12 ! " 4$ & $0^{+0.28}$ \\
\hline $9517(\mathrm{C} 2)$ & $2008-06-05$ & ACIS-I3 & $14^{\prime} \cdot 6$ & 99 & $16 . \prime 7$ & $11.1 \pm 0.3$ \\
\hline 12951(C3) & 2011-03-28 & ACIS-S1 & $10^{\prime} .9$ & 74 & 13 .' $4^{\prime}$ & $13.8 \pm 0.4$ \\
\hline $13246(\mathrm{C} 4)$ & 2011-03-30 & ACIS-S1 & $10^{\prime} .9$ & 45 & $13^{\prime \prime} 4$ & $13.9 \pm 0.6$ \\
\hline $13247(\mathrm{C} 5)$ & 2011-03-31 & ACIS-S1 & $10^{\prime} \cdot 9$ & 36 & $13, " 4$ & $14.2 \pm 0.6$ \\
\hline 12952(C6) & 2011-04-05 & ACIS-S1 & $10^{\prime} .9$ & 143 & $13 .{ }^{\prime \prime} 4$ & $13.4 \pm 0.3$ \\
\hline 12953(C7) & 2011-04-07 & ACIS-S1 & $10^{\prime} .9$ & 32 & $13^{\prime \prime} 4$ & $15.3 \pm 0.7$ \\
\hline $13253(\mathrm{C} 8)$ & 2011-04-08 & ACIS-S1 & $10^{\prime} .9$ & 118 & 13 I. $4^{\prime}$ & $13.8 \pm 0.3$ \\
\hline 13255(C9) & 2011-04-10 & ACIS-S1 & $10^{\prime} 9$ & 43 & $13 ! \prime 4$ & $13.3 \pm 0.6$ \\
\hline 17019(C10) & $2015-02-23$ & ACIS-S3 & 0.3 & 37 & $1 . .6$ & $4.9 \pm 0.4$ \\
\hline \multicolumn{7}{|l|}{ Swift: } \\
\hline $00033207001(\mathrm{~S} 1)$ & $2014-03-28$ & XRT & $1 ! 0$ & 8.7 & $25^{\prime \prime}$ & $1.9 \pm 0.5$ \\
\hline $00033207002(\mathrm{~S} 2)$ & 2016-02-03 & XRT & $3^{\prime} .8$ & 3.1 & $25^{\prime \prime}$ & \\
\hline $00033207003(\mathrm{~S} 3)$ & 2016-02-04 & XRT & $2 \cdot 0$ & 0.3 & $25^{\prime \prime}$ & $7^{+1.3}$ \\
\hline $00033207004(\mathrm{~S} 4)$ & 2016-02-05 & XRT & 1.5 & 2.7 & $25^{\prime \prime}$ & $0.7^{+1.3}$ \\
\hline 00033207005 (S5) & 2016-02-07 & XRT & $1 \ldots 3$ & 2.9 & $25^{\prime \prime}$ & \\
\hline $00033207007(\mathrm{~S} 6)$ & 2016-02-10 & XRT & $1 \% 2$ & 3.0 & $25^{\prime \prime}$ & \\
\hline $00033207008(\mathrm{~S} 7)$ & $2016-02-12$ & XRT & $1 \frac{\prime}{0} 0$ & 2.3 & $25^{\prime \prime}$ & $2.8 \pm 0.7$ \\
\hline 00033207009 (S8) & 2016-02-14 & XRT & $1^{\prime} .4$ & 1.4 & $25^{\prime \prime}$ & \\
\hline
\end{tabular}


Supplementary Table 2 | Spectral fit results. Columns : (1) the observations; (2) the model; (3) intrinsic absorption column density; (4) the apparent inner disk temperature of the MCD; (5) the MCD normization $N_{\mathrm{MCD}} \equiv\left(\left(R_{\mathrm{MCD}} / \mathrm{km}\right) /(D / 10 \mathrm{kpc})\right)^{2} \cos \theta$, where $R_{\mathrm{MCD}}$ is the apparent inner disk radius, $D$ is the source distance, $\theta$ is the disk inclination; (6) the PL photon index; (7) the PL normalization; (8) the reduced $\chi^{2}$ and degrees of freedom for fits using the $\chi^{2}$ statistic (we rebinned the spectra to have at least 20 counts in each bin in order to use the $\chi^{2}$ statistic; other fits without reduced $\chi^{2}$ used the $\mathrm{C}$ statistic); (9) the $0.34-11.5 \mathrm{keV}$ luminosity, corrected for the Galactic absorption but not instrinsic absorption (10) the 0.34-11.5 keV luminosity (the rest frame), corrected for the Galactic absorption and intrinsic absorption. The fits used spectra within 0.3-10 keV for XMM-Newton and Swift and used spectra within 0.3-7 keV for Chandra. Parameters without errors were fixed in the fits. All errors are at the $90 \%$-confidence level.

\begin{tabular}{|c|c|c|c|c|c|c|c|c|c|}
\hline $\begin{array}{r}\text { Observation } \\
\text { (1) }\end{array}$ & $\begin{array}{c}\text { model } \\
(2)\end{array}$ & $\begin{array}{c}N_{\mathrm{H}} \\
\left(10^{22} \mathrm{~cm}^{-2}\right) \\
(3)\end{array}$ & $\begin{array}{c}k T_{\mathrm{MCD}} \\
(\mathrm{keV}) \\
(4)\end{array}$ & $\begin{array}{c}N_{\mathrm{MCD}} \\
(5)\end{array}$ & $\begin{array}{c}\Gamma_{\mathrm{PL}} \\
(6)\end{array}$ & $\begin{array}{c}N_{\mathrm{PL}} \\
\left(10^{-6}\right) \\
(7)\end{array}$ & $\begin{array}{c}\chi_{\nu}^{2}(\nu) \\
(8)\end{array}$ & \multicolumn{2}{|c|}{$\left(10^{43} \mathrm{erg} \mathrm{s}^{-1}\right)$} \\
\hline \multirow{3}{*}{$\mathrm{X} 1$} & $\mathrm{MCD}+\mathrm{PL}$ & 0.42 & $0.10_{-0.04}^{+0.06}$ & $210_{-204}^{+27841}$ & 2.5 & $4.6_{-3.4}^{+3.9}$ & $\cdots$ & $0.08_{-0.03}^{+0.04}$ & $0.55_{-0.31}^{+0.95}$ \\
\hline & MCD & $0.00_{0.00}^{+0.17}$ & $0.32_{-0.15}^{+0.04}$ & $0.08_{-0.07}^{+0.93}$ & $\cdots$ & $\begin{array}{l}-3.4 \\
\ldots\end{array}$ & $\ldots$ & $0.06_{-0.02}^{+0.02}$ & $0.06_{-0.02}^{+0.05}$ \\
\hline & PL & $0.01_{-0.01}^{+0.40}$ & $\ldots$ & $\ldots$ & $2.71_{-0.68}^{+2.34}$ & $4.4_{-1.8}^{+7.2}$ & $\ldots$ & $0.08_{-0.04}^{+0.04}$ & $0.08_{-0.03}^{+0.51}$ \\
\hline \multirow{3}{*}{$\mathrm{x} 2$} & $\mathrm{MCD}+\mathrm{PL}$ & $0.24_{-0.05}^{+0.05}$ & $0.29_{-0.03}^{+0.03}$ & $5.67_{-2.25}^{+4.11}$ & 2.5 & $14.8_{-11.6}^{+11.4}$ & $1.03(105)$ & $1.22_{-0.07}^{+0.07}$ & $2.66_{-0.42}^{+0.51}$ \\
\hline & MCD & $0.21_{-0.04}^{+0.05}$ & $0.31_{-0.02}^{+0.02}$ & $3.94_{-1.27}^{+1.25}$ & $\ldots$ & -11.0 & $1.06(106)$ & $1.17_{-0.06}^{+0.06}$ & $2.31_{-0.27}^{+0.34}$ \\
\hline & $\mathrm{PL}$ & $0.64_{-0.07}^{+0.08}$ & $\ldots$ & $\ldots$ & $4.93_{-0.28}^{+0.30}$ & $464_{-71}^{+94}$ & $1.17(106)$ & $1.19_{-0.06}^{+0.06}$ & $25_{-7}^{+12}$ \\
\hline \multirow{3}{*}{ X3 } & $\mathrm{MCD}+\mathrm{PL}$ & $0.20_{-0.05}^{+0.05}$ & $0.28_{-0.03}^{+0.03}$ & $4.93_{-2.09}^{+4.19}$ & 2.5 & $19.3_{-11.9}^{+11.7}$ & $0.88(98)$ & $1.19_{-0.07}^{+0.07}$ & $2.35_{-0.38}^{+0.47}$ \\
\hline & MCD & $0.16_{-0.04}^{+0.04}$ & $0.32_{-0.02}^{+0.02}$ & $2.91_{-0.96}^{+1.49}$ & $\ldots$ & $\ldots$ & $0.94(99)$ & $1.12_{-0.05}^{+0.05}$ & $1.93_{-0.22}^{+0.27}$ \\
\hline & PL & $0.55_{-0.06}^{+0.07}$ & $\ldots$ & $\ldots$ & $4.69_{-0.27}^{+0.29}$ & $359_{-50}^{+61}$ & $0.91(99)$ & $1.15_{-0.06}^{+0.06}$ & $16_{-5}^{+7}$ \\
\hline \multirow{3}{*}{$\mathrm{C} 2$} & $\mathrm{MCD}+\mathrm{PL}$ & $0.15_{-0.11}^{+0.14}$ & $0.32_{-0.04}^{+0.05}$ & $2.22_{-1.22}^{+3.32}$ & 2.5 & $9.9_{-9.9}^{+10.3}$ & $0.98(42)$ & $1.13_{-0.13}^{+0.17}$ & $1.84_{-0.43}^{+0.67}$ \\
\hline & MCD & $0.09_{-0.09}^{+0.11}$ & $0.35_{-0.04}^{+0.04}$ & $1.43_{-0.64}^{+1.22}$ & .. & -9.9 & $1.01(43)$ & $1.14_{-0.14}^{+0.13}$ & $1.55_{-0.39}^{+0.49}$ \\
\hline & $\mathrm{PL}$ & $0.72_{-0.16}^{+0.18}$ & $\ldots$ & 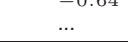 & $5.02_{-0.40}^{+0.47}$ & $471_{-116}^{+185}$ & $0.92(43)$ & $1.02_{-0.10}^{+0.11}$ & $27_{-12}^{+27}$ \\
\hline \multirow{3}{*}{ C3-C9 } & $\mathrm{MCD}+\mathrm{PL}$ & $0.14_{-0.03}^{+0.03}$ & $0.34_{-0.02}^{+0.03}$ & $0.80_{-0.22}^{+0.34}$ & 2.5 & $14.5_{-3.6}^{+3.7}$ & $0.97(137)$ & $0.64_{-0.02}^{+0.02}$ & $1.00_{-0.08}^{+0.09}$ \\
\hline & MCD & $0.07_{-0.02}^{+0.03}$ & $0.40_{-0.02}^{+0.02}$ & $0.39_{-0.08}^{+0.10}$ & $\ldots$ & $=-3.0$ & $1.25(138)$ & $0.60_{-0.02}^{+0.02}$ & $0.74_{-0.04}^{+0.04}$ \\
\hline & $\mathrm{PL}$ & $0.47_{-0.04}^{+0.04}$ & 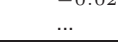 & $\begin{array}{l}-0.08 \\
\ldots\end{array}$ & $4.09_{-0.13}^{+0.13}$ & $147_{-12}^{+13}$ & $0.86(138)$ & $0.61_{-0.02}^{+0.02}$ & $4.57_{-0.62}^{+0.04}$ \\
\hline \multirow{3}{*}{$\mathrm{C} 10$} & $\mathrm{MCD}+\mathrm{PL}$ & $0.46_{-0.23}^{+0.27}$ & $0.13_{-0.02}^{+0.03}$ & $461_{-411}^{+4600}$ & 2.5 & $2.3_{-1.3}^{+1.8}$ & $\cdots$ & $0.31_{-0.05}^{+0.06}$ & $3.52_{-2.31}^{+9.45}$ \\
\hline & MCD & $0.20_{-0.18}^{+0.21}$ & $0.17_{-0.03}^{+0.04}$ & $27_{-22}^{+158}$ & $\ldots$ & $\ldots$ & $\ldots$ & $0.32_{-0.06}^{+0.10}$ & $0.97_{-0.53}^{+1.51}$ \\
\hline & $\mathrm{PL}$ & $0.81_{-0.27}^{+0.32}$ & $\ldots$ & $\cdots$ & $8.01_{-1.42}^{+0.99}$ & $136_{-63}^{+134}$ & $\ldots$ & $0.31_{-0.05}^{+0.06}$ & $96_{-81}^{+688}$ \\
\hline
\end{tabular}



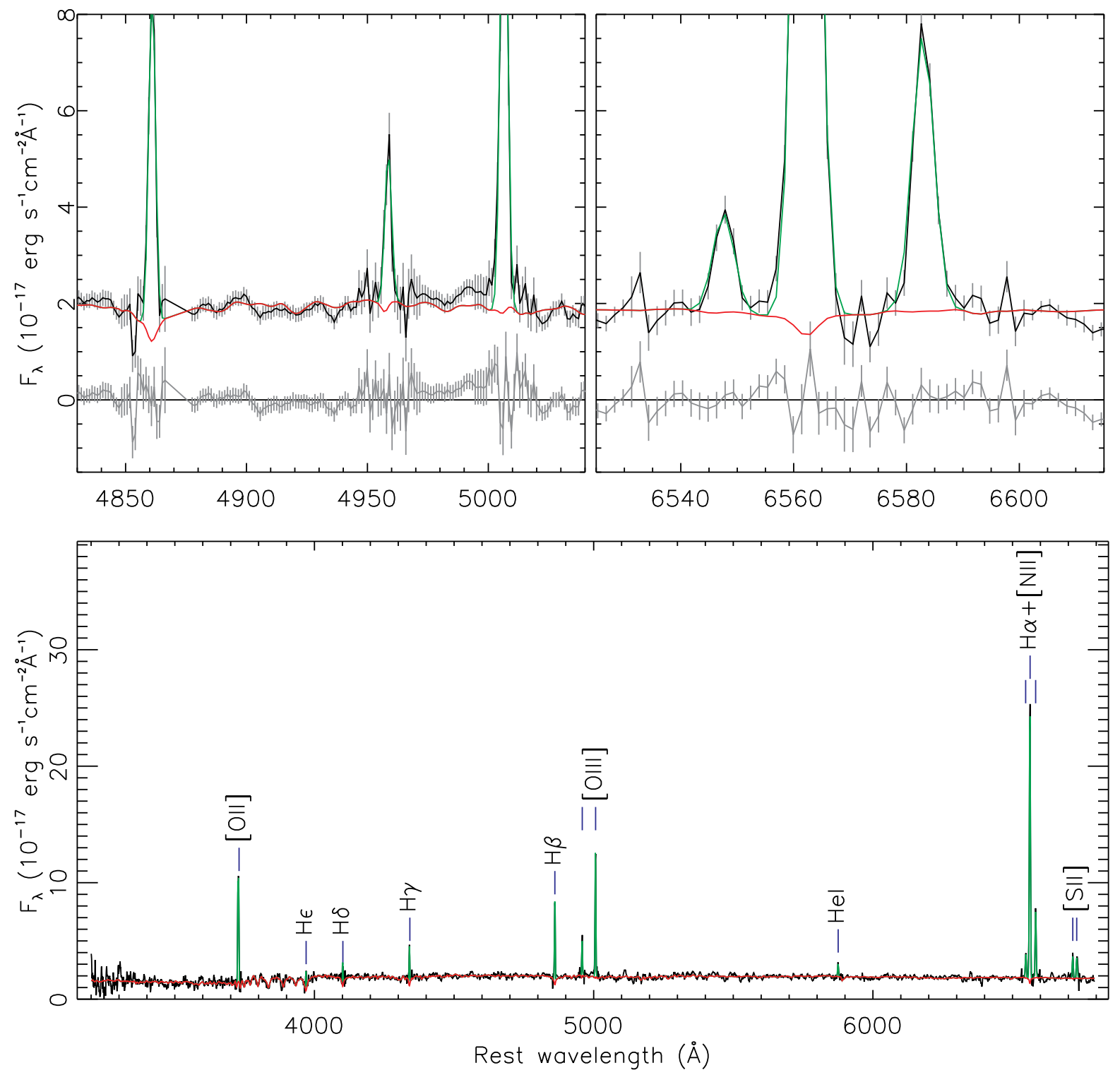

Supplementary Figure 1 | The SDSS optical spectrum of the candidate host galaxy of XJ1500+0154 taken on March 3rd 2011, showing only narrow emission lines consistent with a starburst galaxy. The upper two panels zoom into the $\mathrm{H} \beta-[\mathrm{O}$ III] and $\mathrm{H} \alpha$-[N II] regions, with the fit residuals. The pPXF fit is shown as a solid green line, while the star component is shown as a red line. The data points outside the emission line regions have been smoothed with a box function of width 5 , for clarity. 


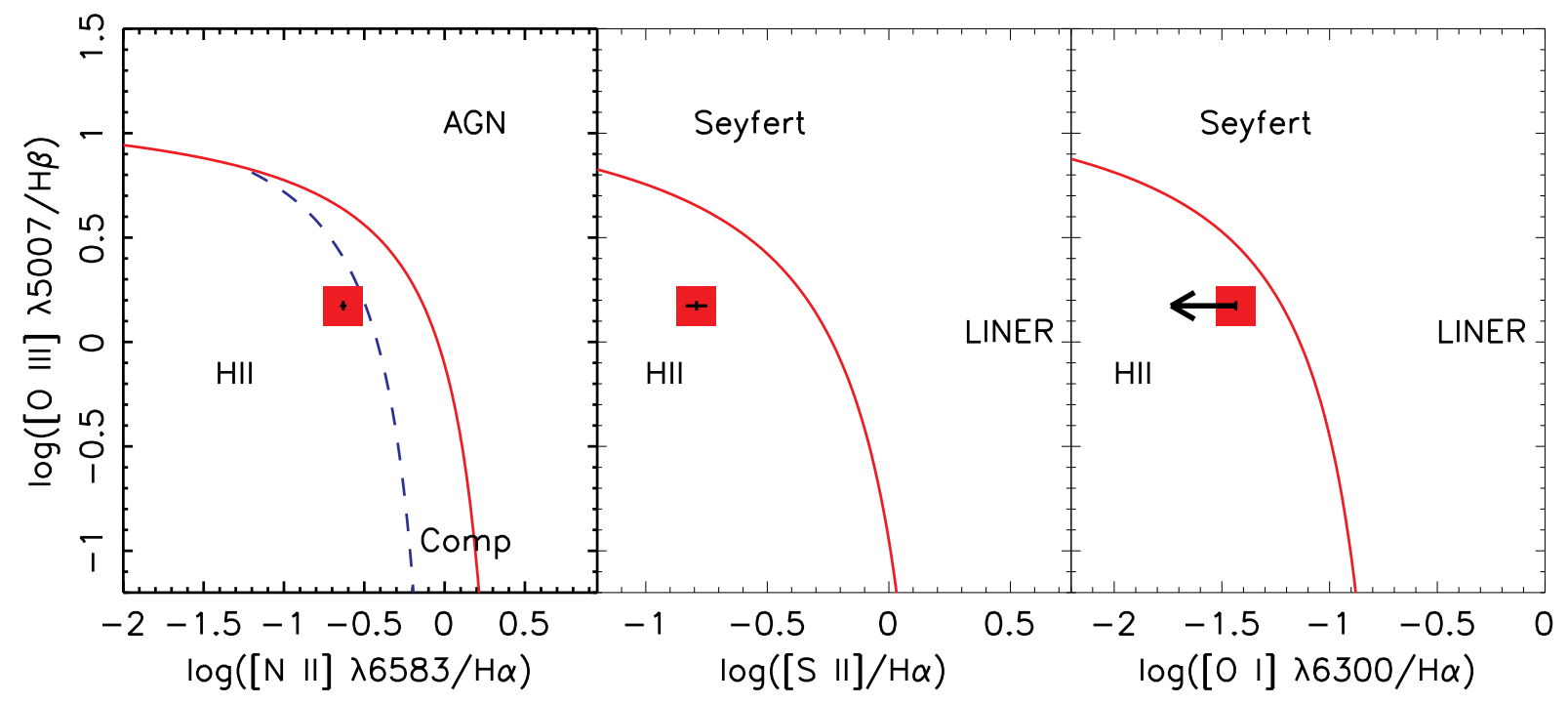

Supplementary Figure $2 \mid \mathbf{X J 1 5 0 0 + 0 1 5 4}$ on the BPT diagrams, indicating it as a star-forming galaxy. The dashed and solid lines are used to separate galaxies into HII-region-like, AGN, and composite types 114 


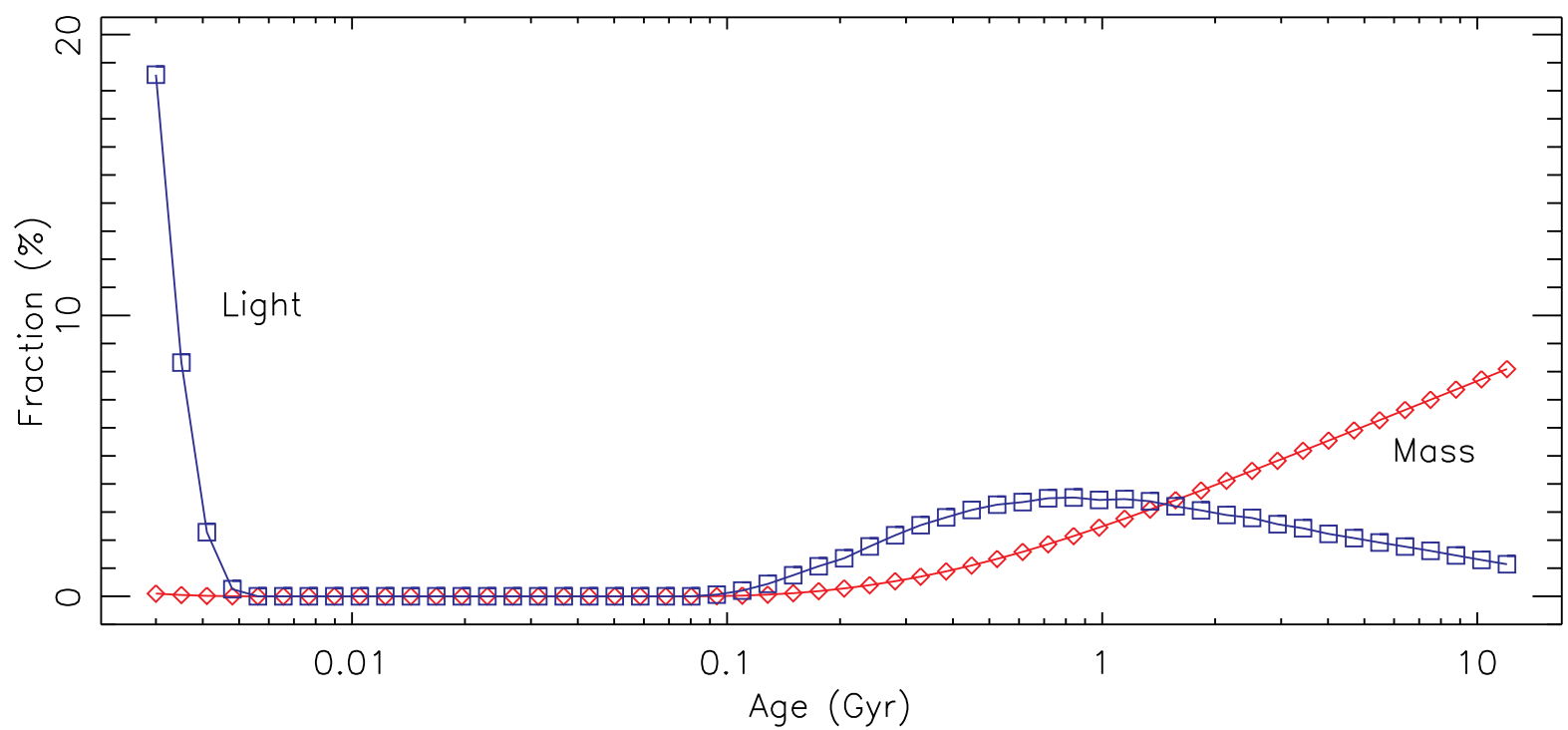

Supplementary Figure 3 The distribution of the mass and light with respect to the age from the pPXF fit to the SDSS optical spectrum of the candidate host galaxy of XJ1500+0154, indicating the presence of very young ( $<5$ Myr) populations. The light was integrated over source rest-frame $3200 \AA$ and $6800 \AA$. 


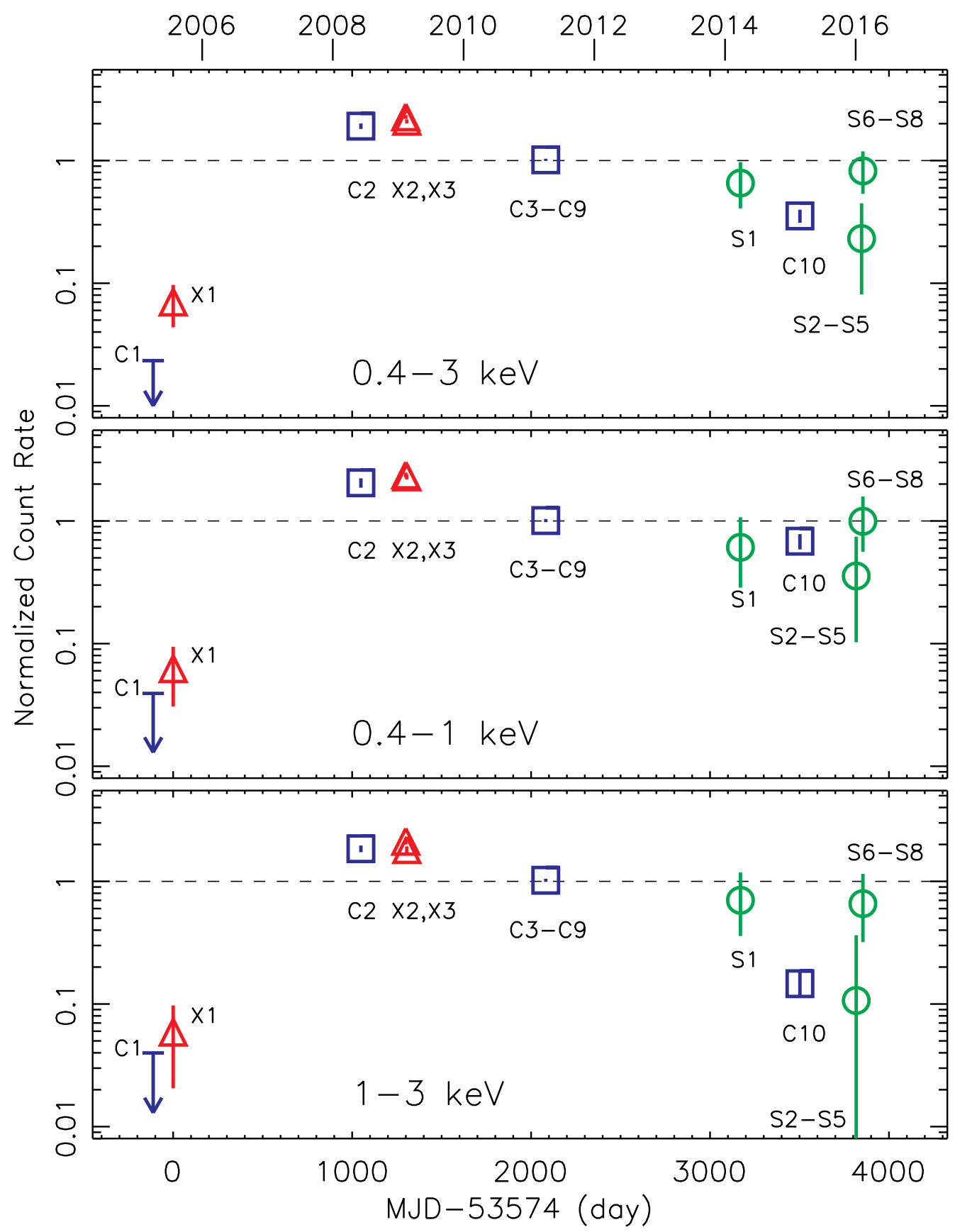

Supplementary Figure $4 \mid$ The long-term evolution of the count rates in 0.4-3 keV, 0.4-1 keV and 1-3 keV (observer frame), normalized to those expected assuming the CompTT fit to C3-C9, with 90\% errors or $3 \sigma$ upper limit (for C1). For XMM-Newton observations, the normalized count rates are the mean of all available cameras weighted by the error. 

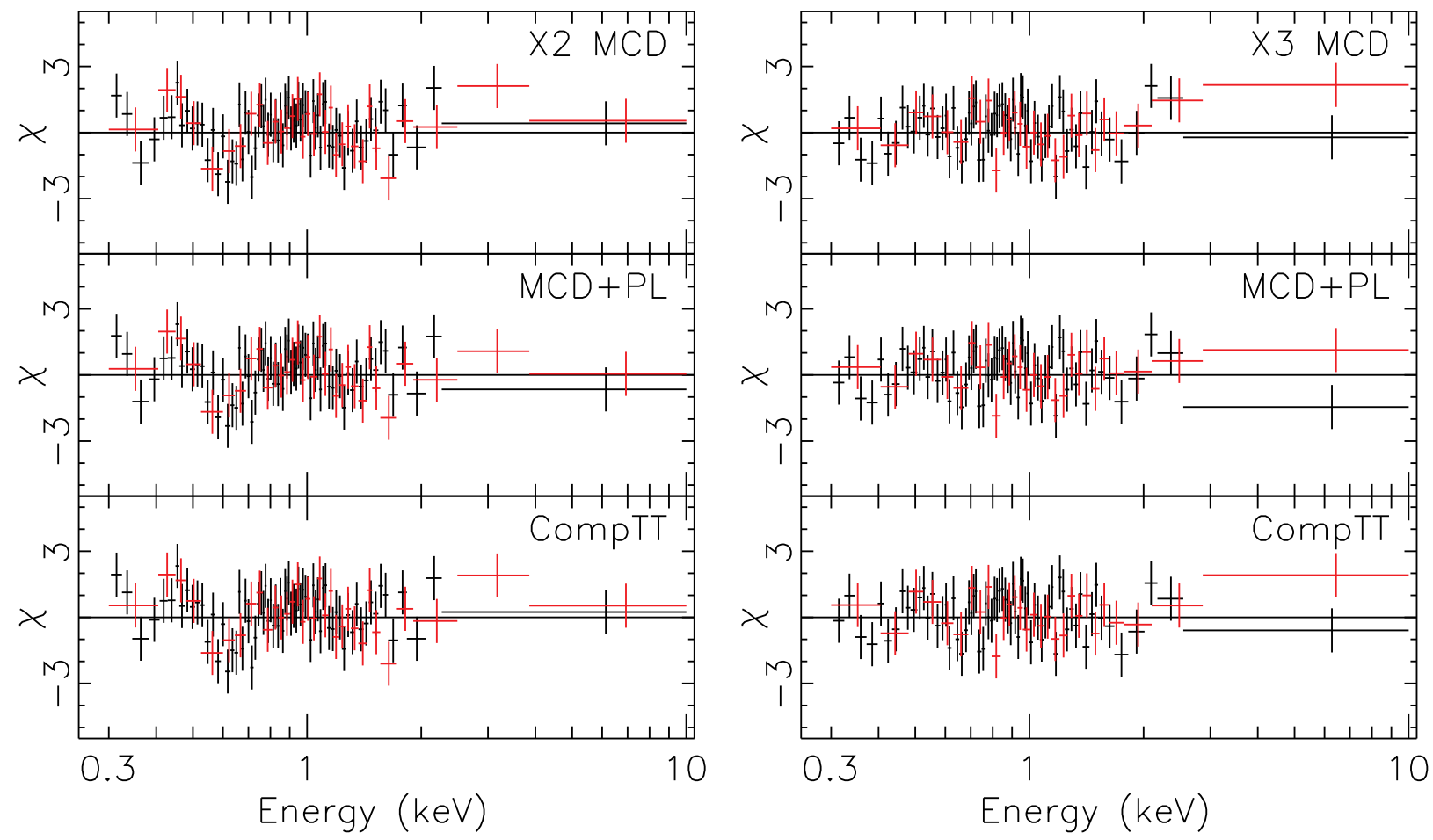

(a)

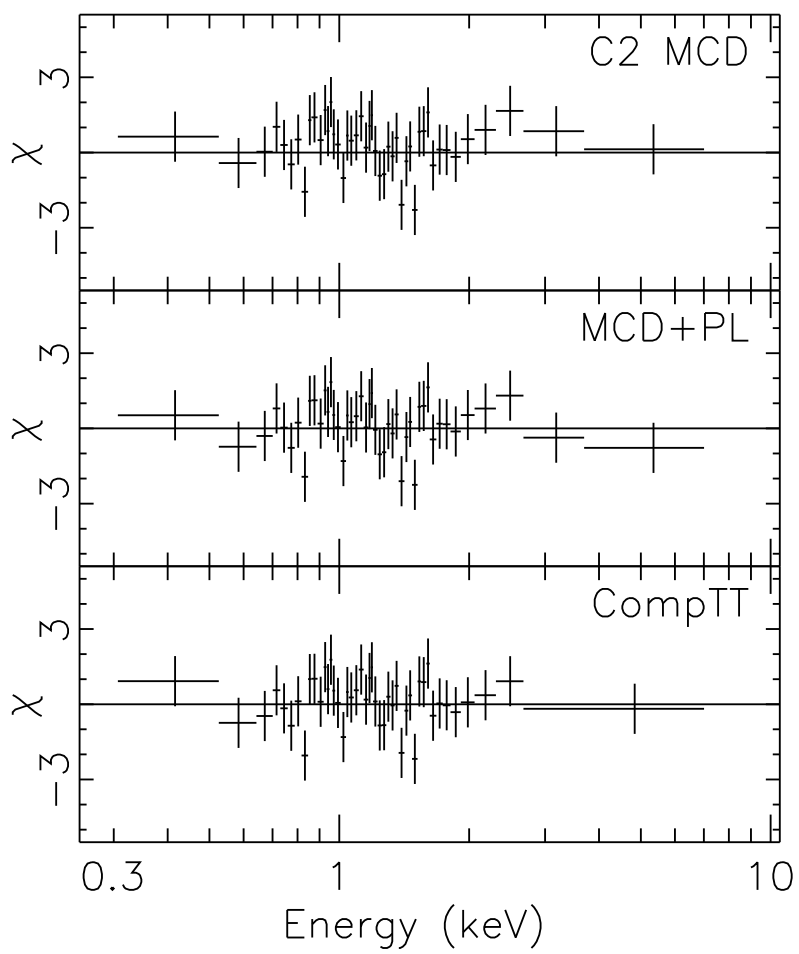

(c)

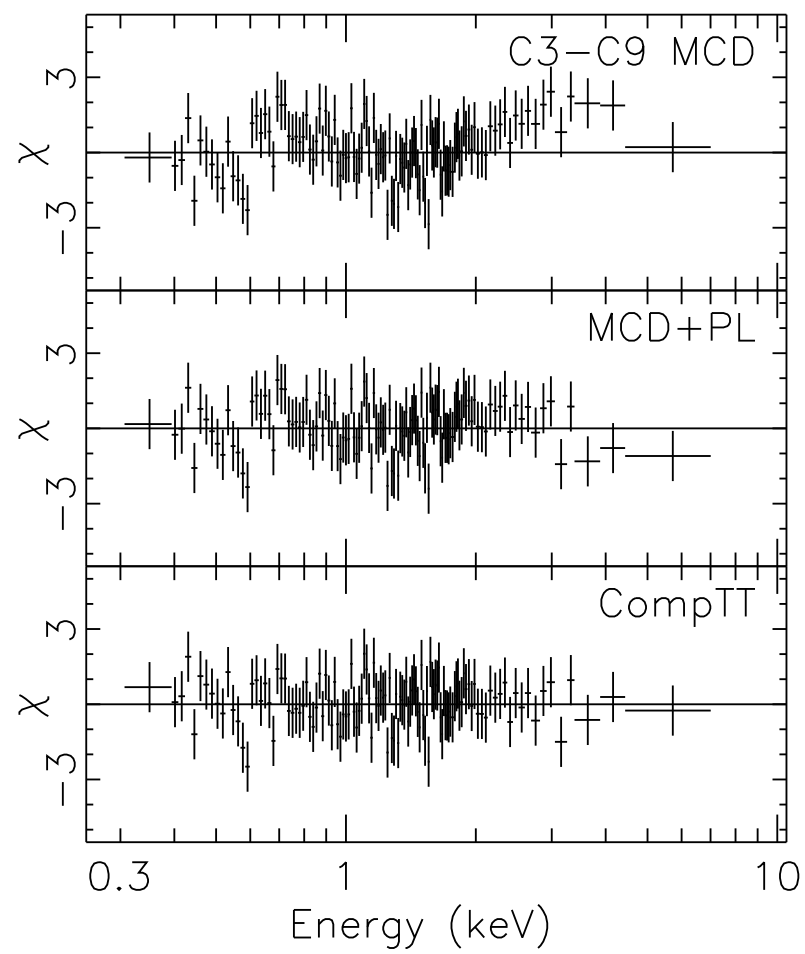

(d)

Supplementary Figure 5 | The fit residuals for the X2, X3, C2, and C3-C9 spectra, with the MCD, MCD+PL, and CompTT models. 


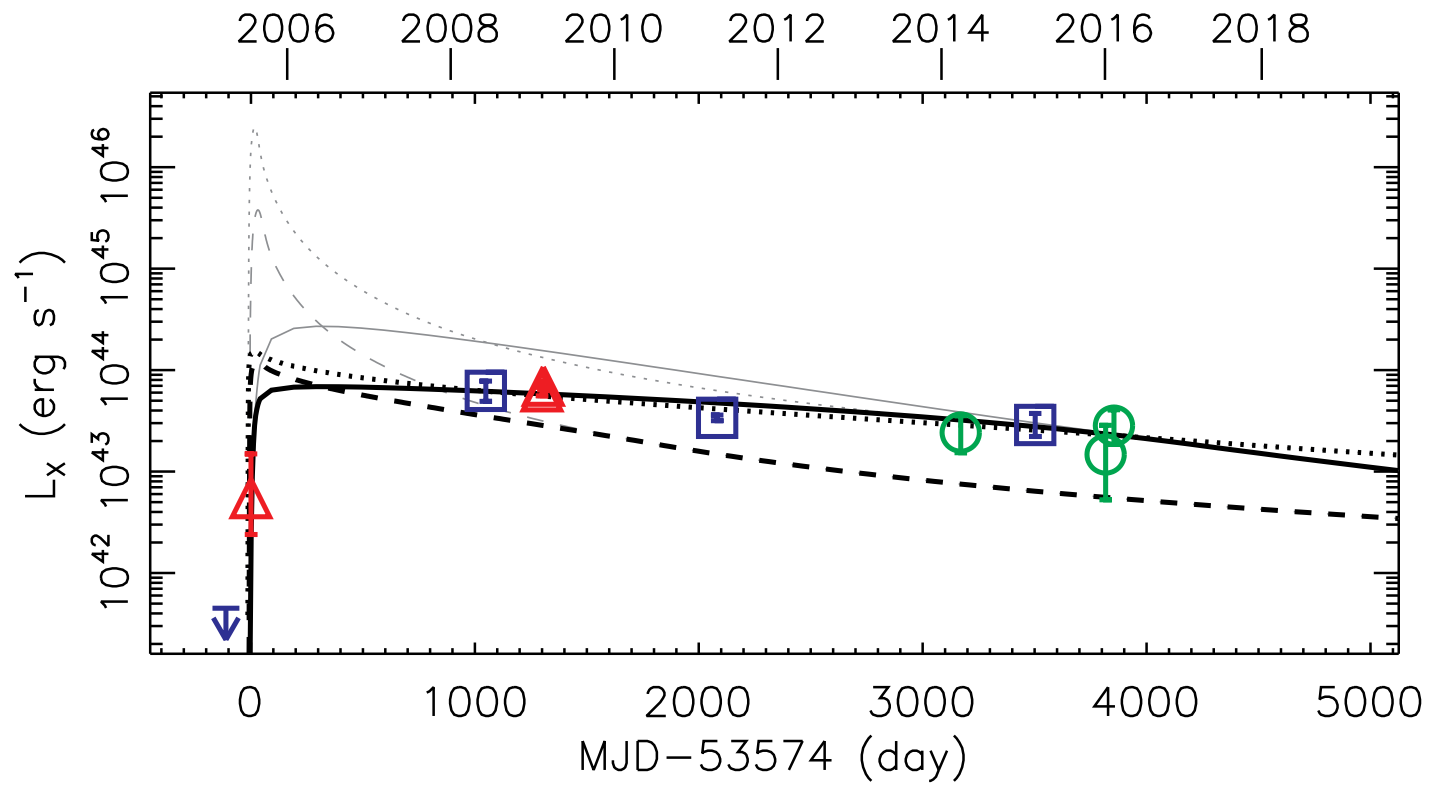

Supplementary Figure 6 | The modeling of the long-term X-ray luminosity curve of XJ1500+0154. The meanings of the symbols are the same as in Figure 2 The lines are for different models: solid lines for a $2 M_{\odot}$ star with slow circularization, dashed lines for a $2 M_{\odot}$ star with prompt circularization, and dotted lines for a $10 M_{\odot}$ star with prompt circularization. The thick black lines incorporate the super-Eddington accretion effects, while the thin gray lines do not (see $S I$ ); they deviate from each other when the accretion rate is super-Eddington. All models assume a SMBH of mass $10^{6} M_{\odot}$. 

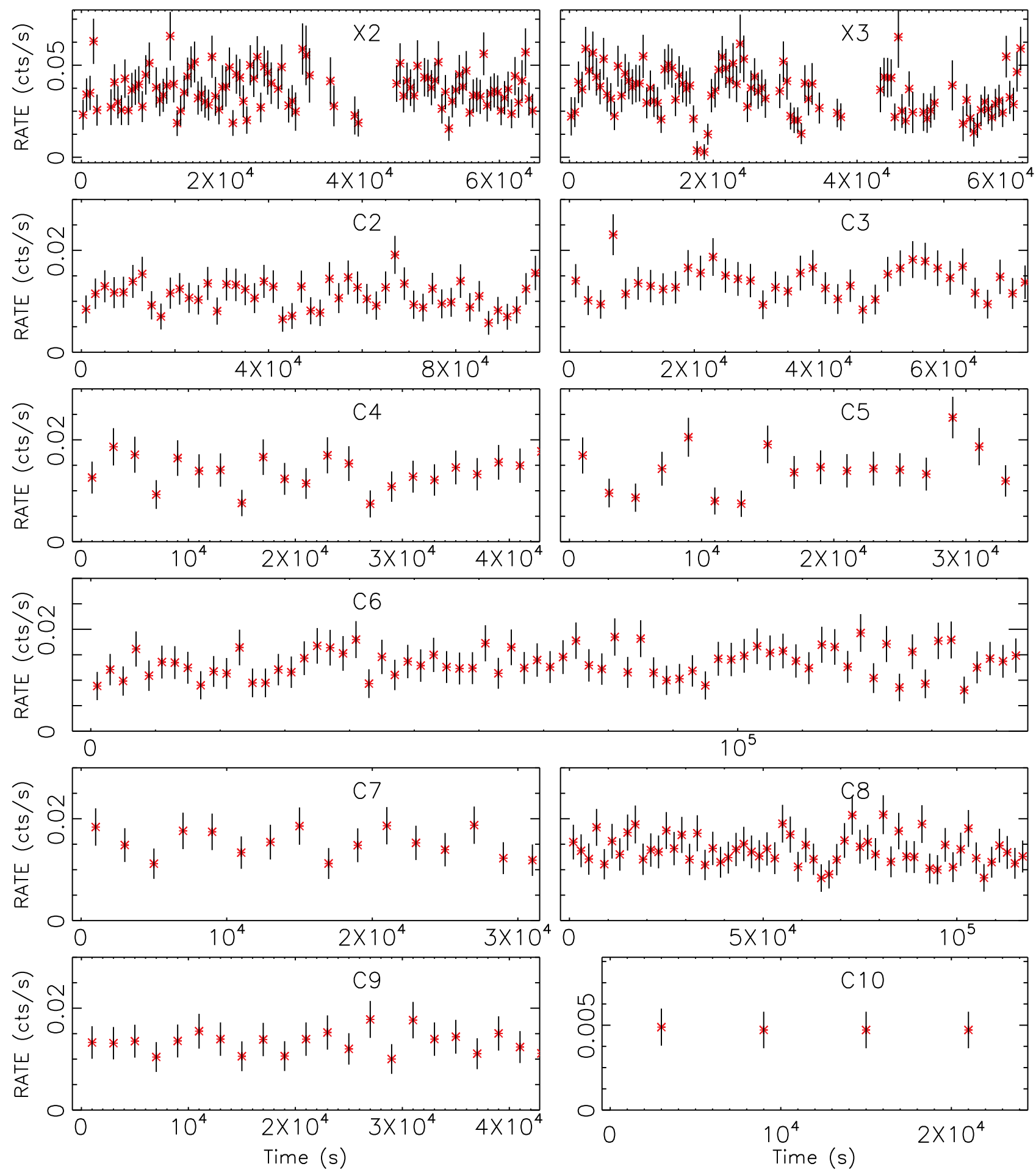

Supplementary Figure 7 The background subtracted light curves from bright observations (0.3-3 keV for XMM-Newton observations and 0.4-3 keV for Chandra observations). For X2 and X3, we only show the pn data for clarity, and data in the strong background intervals have been excluded. The light curve bin size is $500 \mathrm{~s}$ for $\mathrm{X} 2$ and $\mathrm{X} 3,2 \mathrm{ks}$ for $\mathrm{C} 2-\mathrm{C} 9$, and $6 \mathrm{ks}$ for $\mathrm{C} 10$. There seems to be a fast drop to zero count rate at $20 \mathrm{ks}$ into the $\mathrm{X} 3$ observation. 


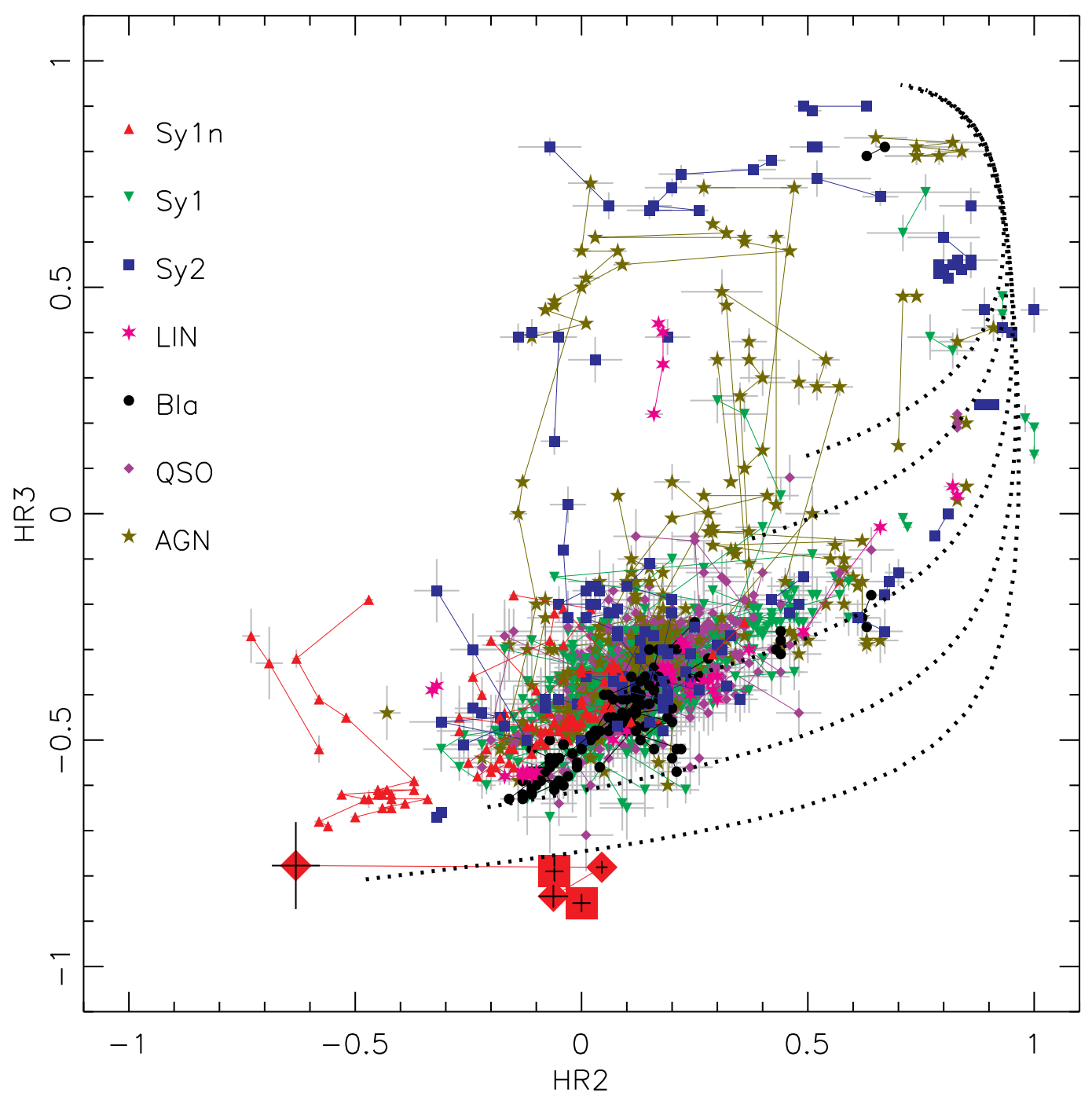

Supplementary Figure 8 | The X-ray color-color diagram for identified AGNs in Lin et al. 2012 ${ }^{78}$ and XJ1500+0154 (big red squares) from the 3XMM-DR5 catalog. The colors HR2 and HR3 are defined using $(H-S) /(H+S)$, with $S$ and $H$ being the MOS1-medium-filter equivalent $0.5-1 \mathrm{keV}$ and $1-2 \mathrm{keV}$ counts rates for HR2 and 1-2 keV and 2-4.5 keV count rates for HR3, respectively. The Chandra C2, C3-C9 and C10 observations are shown as big red diamonds. We overplot PL spectra (dotted lines) with $\Gamma_{\mathrm{PL}}=0.5$ (top), $1,2,3$, and 4 and $N_{\mathrm{H}}$ varying from 0 (lower-left) to $10^{23} \mathrm{~cm}^{-2}$. The detections for each source are connected by solid lines in an increasing order of HR3. We only show AGN detections with $\mathrm{S} / \mathrm{N} \geq 18$ (based on the $0.2-4.5 \mathrm{keV}$ flux), resulting in 2002 detections in total. XJ1500+0154 has lower values of HR3 (thus softer in 1-4.5 keV) than AGNs in all observations shown. 
52. Cappellari, M. \& Emsellem, E. Parametric Recovery of Line-of-Sight Velocity Distributions from Absorption-Line Spectra of Galaxies via Penalized Likelihood. Publ. Astron. Soc. Pacif. 116, 138-147 (2004). astro-ph/0312201

53. Maraston, C. \& Strömbäck, G. Stellar population models at high spectral resolution. Mon. Not. R. Astron. Soc. 418, 2785-2811 (2011). 1109.0543

54. Prugniel, P. \& Soubiran, C. A database of high and mediumresolution stellar spectra. Astron. Astrophys. 369, 1048-1057 (2001). astro-ph/0101378

55. Maraston, C., Strömbäck, G., Thomas, D., Wake, D. A. \& Nichol, R. C Modelling the colour evolution of luminous red galaxies - improvements with empirical stellar spectra. Mon. Not. R. Astron. Soc. 394, L107-L111 (2009). 0809.1867

56. Schlegel, D. J., Finkbeiner, D. P. \& Davis, M. Maps of Dust Infrared Emission for Use in Estimation of Reddening and Cosmic Microwave Background Radiation Foregrounds. Astrophys. J. 500, 525-+ (1998). arXiv:astro-ph/9710327

57. Baldwin, J. A., Phillips, M. M. \& Terlevich, R. Classification parameters for the emission-line spectra of extragalactic objects. Publ. Astron. Soc. Pacif. 93, 5-19 (1981).

58. Veilleux, S. \& Osterbrock, D. E. Spectral classification of emission-line galaxies. Astrophys. J. Suppl. Ser. 63, 295-310 (1987)

59. Kennicutt, R. C., Jr. Star Formation in Galaxies Along the Hubble Sequence. Annu. Rev. Astron. Astrophys. 36, 189-232 (1998). astro-ph/9807187

60. Komossa, S., Xu, D., Zhou, H., Storchi-Bergmann, T. \& Binette, L. On the Nature of Seyfert Galaxies with High [O III] $\lambda 5007$ Blueshifts. Astrophys. J. 680, 926-938 (2008). 0803.0240

61. Mullaney, J. R. et al. Narrow-line region gas kinematics of 24264 optically selected AGN: the radio connection. Mon. Not. R. Astron. Soc. 433 622-638 (2013). 1305.0263

62. Harrison, C. M., Alexander, D. M., Mullaney, J. R. \& Swinbank, A. M. Kiloparsec-scale outflows are prevalent among luminous AGN: outflows and feedback in the context of the overall AGN population. Mon. Not. R. Astron. Soc. 441, 3306-3347 (2014). 1403.3086

63. Rupke, D. S., Veilleux, S. \& Sanders, D. B. Outflows in Infrared-Luminous Starbursts at z ; 0.5. II. Analysis and Discussion. Astrophys. J. Suppl. Ser. 160, 115-148 (2005). astro-ph/0506611

64. Holoien, T. W.-S. et al. Six Months of Multi-Wavelength Follow-up of the Tidal Disruption Candidate ASASSN-14li and Implied TDE Rates from ASAS-SN. ArXiv e-prints (2015). 1507.01598

65. Saxton, R. D. et al. A tidal disruption-like X-ray flare from the quiescent galaxy SDSS J120136.02+300305.5. Astron. Astrophys. 541, A106 (2012). 1202.5900

66. Remillard, R. A. \& McClintock, J. E. X-Ray Properties of BlackHole Binaries. Annu. Rev. Astron. Astrophys. 44, 49-92 (2006). astro-ph/0606352

67. Done, C., Gierliński, M. \& Kubota, A. Modelling the behaviour of accretion flows in X-ray binaries. Everything you always wanted to know about accretion but were afraid to ask. Astron. Astrophys. Rev 15, 1-66 (2007). 0708.0148

68. Done, C., Davis, S. W., Jin, C., Blaes, O. \& Ward, M. Intrinsic disc emission and the soft X-ray excess in active galactic nuclei. Mon. Not. R. As tron. Soc. 420, 1848-1860 (2012). 1107.5429

69. Alexander, K. D., Berger, E., Guillochon, J., Zauderer, B. A. \& Williams, P. K. G. Discovery of an Outflow from Radio Observations of the Tidal Disruption Event ASASSN-14li. Astrophys. J. 819, L25 (2016). 1510.01226

70. Lin, D. et al. An Ultrasoft X-Ray Flare from 3XMM J152130.7+074916: A Tidal Disruption Event Candidate. Astrophys. J. 811, 43 (2015). 1509.00840

71. Guillochon, J. \& Ramirez-Ruiz, E. Hydrodynamical Simulations to Determine the Feeding Rate of Black Holes by the Tidal Disruption of Stars: The Importance of the Impact Parameter and Stellar Structure. Astrophys. J. 767, 25 (2013). 1206.2350

72. Lin, D., Remillard, R. A. \& Homan, J. Spectral States of XTE J1701-462 Link Between Z and Atoll Sources. Astrophys. J. 696, 1257-1277 (2009). 0901.0031

73. MacLeod, M., Guillochon, J. \& Ramirez-Ruiz, E. The Tidal Disruption of Giant Stars and their Contribution to the Flaring Supermassive Black Hole Population. Astrophys. J. 757, 134 (2012). 1206.2922

74. MacLeod, M., Ramirez-Ruiz, E., Grady, S. \& Guillochon, J. Spoonfeeding Giant Stars to Supermassive Black Holes: Episodic Mass Transfer from Evolving Stars and their Contribution to the Quiescent Activity of Galactic Nuclei. Astrophys. J. 777, 133 (2013). 1307.2900
75. Voges, W. et al. The ROSAT all-sky survey bright source catalogue. Astron. Astrophys. 349, 389-405 (1999). arXiv: astro-ph/9909315

76. Holoien, T. W.-S. et al. ASASSN-15oi: A Rapidly Evolving, Luminous Tidal Disruption Event at $216 \mathrm{Mpc}$. ArXiv e-prints (2016). 1602.01088

77. Lamastra, A. et al. The bolometric luminosity of type 2 AGN from extinction-corrected [OIII]. No evidence of Eddington-limited sources. Astron. Astrophys. 504, 73-79 (2009). 0905.4439

78. Lin, D., Webb, N. A. \& Barret, D. Classification of X-Ray Sources in the XMM-Newton Serendipitous Source Catalog. Astrophys. J. 756, 27 (2012). 1207.1913

79. Grupe, D. et al. Strong UV and X-Ray Variability of the Narrow Line Seyfert 1 Galaxy WPVS 007: on the Nature of the X-Ray Low State. Astron. J. 146, 78 (2013). 1307.7713

80. Terashima, Y., Kamizasa, N., Awaki, H., Kubota, A. \& Ueda, Y. A Candidate Active Galactic Nucleus with a Pure Soft Thermal X-Ray Spectrum. Astrophys. J. 752, 154 (2012). 1205.2774

81. Ho, L. C., Kim, M. \& Terashima, Y. The Low-mass, Highly Accreting Black Hole Associated with the Active Galactic Nucleus 2XMM J123103.2+110648. Astrophys. J. 759, L16 (2012). 1210.0440

82. Lin, D., Irwin, J. A., Godet, O., Webb, N. A. \& Barret, D. A 3.8 hr Periodicity from an Ultrasoft Active Galactic Nucleus Candidate. Astrophys. J. 776, L10 (2013). 1309.4440

83. Lin, D., Webb, N. A. \& Barret, D. Classification of X-Ray Sources in the XMM-Newton Serendipitous Source Catalog: Objects of Special Interest. Astrophys. J. 780, 39 (2014). 1309.0509

84. Miniutti, G. et al. A high Eddington-ratio, true Seyfert 2 galaxy candidate: implications for broad-line region models. Mon. Not. R. Astron. Soc. 433 , 1764-1777 (2013). 1305.3284

85. Saxton, R. D., Motta, S. E., Komossa, S. \& Read, A. M. Was the soft X-ray flare in NGC 3599 due to an AGN disc instability or a delayed tidal disruption event? Mon. Not. R. Astron. Soc. 454, 2798-2803 (2015). 1509.05193

86. Grupe, D., Komossa, S. \& Saxton, R. IC 3599 Did It Again: A Second Outburst of the X-Ray Transient Seyfert 1.9 Galaxy. Astrophys. J. 803, L28 (2015). 1504.01389

87. Campana, S. et al. Multiple tidal disruption flares in the active galaxy IC 3599. Astron. Astrophys. 581, A17 (2015). 1502.07184

88. Shappee, B. J. et al. The Man behind the Curtain: X-Rays Drive the UV through NIR Variability in the 2013 Active Galactic Nucleus Outburst in NGC 2617. Astrophys. J. 788, 48 (2014). 1310.2241

89. LaMassa, S. M. et al. The Discovery of the First Changing Look Quasar New Insights Into the Physics and Phenomenology of Active Galactic Nucleus. Astrophys. J. 800, 144 (2015). 1412.2136

90. Parker, M. L. et al. The detection and X-ray view of the changing look AGN HE 1136-2304. Mon. Not. R. Astron. Soc. 461, 1927-1936 (2016). 1606.04955

91. Risaliti, G. et al. The XMM-Newton long look of NGC 1365: uncovering of the obscured X-ray source. Mon. Not. R. Astron. Soc. 393, L1-L5 (2009). 0811.1594

92. Risaliti, G. et al. Variable Partial Covering and A Relativistic Iron Line in NGC 1365. Astrophys. J. 696, 160-171 (2009). 0901.4809

93. Filippenko, A. V. \& Sargent, W. L. W. Discovery of an extremely low luminosity Seyfert 1 nucleus in the dwarf galaxy NGC 4395. Astrophys. J. 342, L11-L14 (1989).

94. Barth, A. J., Ho, L. C., Rutledge, R. E. \& Sargent, W. L. W. POX 52: A Dwarf Seyfert 1 Galaxy with an Intermediate-Mass Black Hole. Astrophys. J. 607, 90-102 (2004). astro-ph/0402110

95. Reines, A. E., Sivakoff, G. R., Johnson, K. E. \& Brogan, C. L. An actively accreting massive black hole in the dwarf starburst galaxy Henize2-10. Nature 470, 66-68 (2011). 1101.1309

96. Pardo, K. et al. X-ray Detected Active Galactic Nuclei in Dwarf Galaxies at $0<1$. ArXiv e-prints (2016). 1603.01622

97. Fabian, A. C. The obscured growth of massive black holes. Mon. Not. R. Astron. Soc. 308, L39-L43 (1999). astro-ph/9908064

98. Silk, J. \& Rees, M. J. Quasars and galaxy formation. Astron. Astrophys. 331, L1-L4 (1998). astro-ph/9801013

99. Komossa, S. Tidal disruption of stars by supermassive black holes: The X-ray view. In European Physical Journal Web of Conferences, vol. 39 of European Physical Journal Web of Conferences, 2001 (2012).

100. Komossa, S. Tidal disruption of stars by supermassive black holes: Status of observations. JHEA 7, 148-157 (2015). 1505.01093

101. Grupe, D., Thomas, H. \& Leighly, K. M. RX J1624.9+7554: a new X-ray transient AGN. Astron. Astrophys. 350, L31-L34 (1999). arXiv:astro-ph/9909101

102. Komossa, S. \& Greiner, J. Discovery of a giant and luminous X-ray outburst from the optically inactive galaxy pair RX J1242.6-1119. Astron. Astrophys. 349, L45-L48 (1999). arXiv: astro-ph/9908216 
103. Komossa, S. \& Bade, N. The giant X-ray outbursts in NGC 5905 and IC 3599:() hfill Follow-up observations and outburst scenarios. Astron. Astrophys. 343, 775-787 (1999). arXiv: astro-ph/9901141

104. Esquej, P. et al. Evolution of tidal disruption candidates discovered by XMM-Newton. Astron. Astrophys. 489, 543-554 (2008). 0807.4452

105. Lin, D. et al. Discovery of an Ultrasoft X-Ray Transient Source in the 2XMM Catalog: A Tidal Disruption Event Candidate. Astrophys. J. $\mathbf{7 3 8}$ 52-+ (2011). 1106.0744

106. Maksym, W. P., Ulmer, M. P. \& Eracleous, M. A Tidal Disruption Flare in A1689 from an Archival X-ray Survey of Galaxy Clusters. Astrophys. J. 722, 1035-1050 (2010). 1008.4140

107. Maksym, W. P., Lin, D. \& Irwin, J. A. RBS 1032: A Tidal Disruption Event in Another Dwarf Galaxy? Astrophys. J. 792, L29 (2014). 1407.2928

108. Donato, D. et al. A Tidal Disruption Event in a nearby Galaxy Hosting an Intermediate Mass Black Hole. Astrophys. J. 781, 59 (2014). 1311.6162

109. Bloom, J. S. et al. A Possible Relativistic Jetted Outburst from a Massive Black Hole Fed by a Tidally Disrupted Star. Science 333, 203- (2011). 1104.3257

110. Burrows, D. N. et al. Relativistic jet activity from the tidal disruption of a star by a massive black hole. Nature 476, 421-424 (2011). 1104.4787

111. Zauderer, B. A. et al. Birth of a relativistic outflow in the unusual $\gamma$ ray transient Swift J164449.3+573451. Nature 476, 425-428 (2011). 1106.3568

112. Cenko, S. B. et al. Swift J2058.4+0516: Discovery of a Possible Second Relativistic Tidal Disruption Flare? Astrophys. J. 753, 77 (2012). 1107.5307

113. Brown, G. C. et al. Swift J1112.2-8238: a candidate relativistic tida disruption flare. Mon. Not. R. Astron. Soc. 452, 4297-4306 (2015). 1507.03582

114. Kewley, L. J., Groves, B., Kauffmann, G. \& Heckman, T. The host galaxies and classification of active galactic nuclei. Mon. Not. R. Astron. Soc. 372, 961-976 (2006). astro-ph/0605681 